\title{
"HONOR, BONUM ET MAGNUM AVERUM". LA MOBILITÀ MEDIEVALE IN UN CASO PROCESSUALE DEL XII SECOLO
}

\section{GABRIELE ARCHETTI}

UDC: $314.745 \cdot 3-054 \cdot 72 " 04 / 14 "$ $347.65 / 68 " 11$

Original scientific paper

Manuscript received: 23. 03. 2016.

Revised manuscript accepted: 20. 04. 2016.

DOI: 10.1484/J.HAM.5.111347

\author{
G. Archetti \\ Università Cattolica del Sacro Cuore \\ Largo Agostino Gemelli, 1 \\ 20123 Milano, Italia \\ gabriele.archetti@unicatt.it
}

The short, medium and long-range mobility is a characteristic feature of the Middle Ages. The examination of the evidence of the witnesses of a lawsuit for hereditary possession, instituted in the second half of the twelfth century in the Lombard context, offers an interesting and unpublished cross-section on a personal and territorially circumscribed story and on the dynamism of men and women to move for the most disparate causes from one place to another. In particular, the events, recorded inside an aristocratic family of "milites", point out the family ties, the dynamics for the control of power, the intrigues of all kinds to get the paternal heritage and the consequent patrilineage development, the relationships with the ecclesiastical and civil institutions, the constant trips on foot, on horseback or through waterway both locally and regionally than international, to arrive to the big centres of the Communes of northern Italy, to the heart of Christianity, in Rome, and to the capital of the Eastern empire, in Constantinople.

Keywords: The Commune, Inheritance, Parish, Bishop, Journey, Monastery, Podestà, Oath, Pilgrimage, Miles Dominus, Lake Iseo, Montisola, Brescia, Franciacorta, Lombardy, Rodengo, Constantinople, Rome

È sempre molto interessante evidenziare la mobilità di persone e cose, seguirne gli spostamenti e gli interessi, anche nel caso di vicende minute e singolari come quella, dolorosa quanto intrigante, che ruota intorno alla figura femminile di un'astuta aristocratica residente sulle colline della Franciacorta, domina Precia, protagonista e poi vittima del suo stesso inganno ${ }^{1}$. Singolare è altresì la straordinaria capacità di spostamento, di libertà di decisione e di passionalità degli uomini e delle donne del medioevo che per l'intensità, l'affetto e persino la violenza con cui manifestavano i loro sentimenti appaiono di una modernità sorprendente, a conferma che l'interesse per il passato non è motivato dal recupero antiquario di fatti o di un tempo che non esistono più, ma perché tali avvenimenti permettono di indagare il vissuto e l'esperienza di altri, lontani e diversi da noi, ma che però restano prossimi per la loro personale e insieme comune umanità.

Il venerdì 30 ottobre 1180, nella loggia rotonda del palazzo episcopale di Brescia, domina Precia, vedova del dominus Baxacaponus di Rodengo, giurava con la mano sul Vangelo confessando al vescovo Giovanni II un peccato tanto grave quanto insolito: quello di aver fatto credere che il piccolo Baxacaponinus fosse suo figlio, mentre in realtà era suo nipote, essendo nato dalla moglie del suo primogenito $\mathrm{Obizo}^{2}$ (fig. 1). Nelle parole della donna ci sono tutti gli elementi per comprendere i contorni degli accadimenti e il rimorso di una

\footnotetext{
${ }^{1}$ Il testo documentario - due pergamene conservate nell'Archivio Capitolare di Reggio Emilia, Custodia di noce, t. III, nr. 25 (Brescia, 30 ottobre 1180) e nr. 32 (Brescia, 29 luglio 1182), uniche due superstiti del fascicolo processuale - è stato pubblicato da O. GuYOTJEANNIN, Les lois du sang et du patrimoine. Un détournement d'héritage dans la noblesse bresciane à la fin du XII siècle, «Mélanges de l'École française de Rome. Moyen Âge - Temps Modernes», 99, 2 (1987), pp. 765-791 che, insieme alla trascrizione, ne ha fornito una prima ampia lettura; qualche ripresa della vicenda, sia pure con sottolineature diverse e con riferimento più preciso all'ambito geografico, è stata fatta da G. ARCHETTI, "Ad suas manus laborant". Proprietà, economia e territorio rurale nelle carte di Rodengo (secc. XI-XV), in San Nicolò di Rodengo. Un monastero di Franciacorta tra Cluny e Monte Oliveto, a cura di G. Spinelli, P.V. Begni Redona, R. Prestini, Brescia 2002, pp. 91-92; IDEM, Abitato e territorio a Ome nel Medioevo, in La terra di Ome in età medievale, a cura di G. Archetti e A. Valsecchi, Associazione archeologica UsPAAA, Brescia 2003, pp. 44-48, 57; ID., "Singulariter in heremo vivere". Forme di vita eremitica nel medioevo della Lombardia orientale, in Il monachesimo in Valle Camonica, Atti della giornata di studio, 31 maggio 2003, Eremo dei Santi Pietro e Paolo di Bienno - Monastero di San Salvatore di Capo di Ponte, Breno 2004, pp. 127-130; ID., Pievi e monasteri in età romanica. L'inquadramento ecclesiastico delle campagne bresciane tra XI e XIII secolo, in Società bresciana e sviluppi del romanico (XI-XIII secolo), Convegno internazionale di studi (Brescia, 9-10 maggio 2002), a cura di G. Andenna, M. Rossi, Milano 2007, p. 196; ID., "Evangelium nuntiare". Chiese, impegno pastorale e forme di religiosità, in A servizio del Vangelo. Il cammino storico dell'evangelizzazione a Brescia, 1. L'età antica e medievale, a cura di G. Andenna, Brescia 2010, pp. 293-294.

2 «In civitate Brixia, in laubia rotunda episcopi, coram domino Johanne Brixiensi episcopo, domina Precia, relicta quondam Baxacaponi, juravit ad sancta Dei evangelia, posita manu supra librum, dicere veritatem et cellare mendacium [...] et inquisita ab ipso episcopo, dixit, quod predictus Baxacaponinus non fuit natus ex se et predicto Baxacapono, quondam viro suo, set, cum uxor Obizonis filii sui pregnans erat ex ipso Baxacaponino, dicit quod simulavit se pregnantem esse, sed in veritate pregnans non erat et, nato isto Baxacaponino, fecit illum duci ad se et Girardinus de Malxello atulit illum et illa accepit eum et finxit, se iacere in pa[...] de eo, cum in veritate illum non peperat» («Nella città di Brescia, nella loggia rotonda dell'episcopio, davanti al vescovo di Brescia dominus Giovanni, domina Precia, vedova del defunto Baxacaponus, giurò sui santi vangeli di Dio, ponendo la mano sul libro, di dire la verità e respingere il falso [...] e interrogata dal presule, disse che il predetto Baxacaponinus non era stato generato da lei e dal predetto Baxaponus, un tempo suo marito, ma, essendo incinta la moglie di suo figlio Obizo dello stesso Baxacaponino, disse che finse di essere gravida, mentre non lo era, e nato il piccolo Baxacaponino, se lo fece portare e Girardino de Malxello andò a prenderlo e lei lo prese fingendo di giacere in parte a lui anche se in verità non lo aveva partorito», GuYotjeannin, Les lois du sang, pp. 779-780). Sulla figura del vescovo di Brescia Giovanni II da Fiumicello (1174-1195), di cui però non si fa cenno di questa vicenda processuale, cfr. F. SAVIO, Gli antichi vescovi d'Italia dalle origini al 1300 descritti per regioni, I. La Lombardia, parte II: Bergamo, Brescia, Como, Bergamo 1929, pp. 233-239; A. Fappani, F. Trovati, I vescovi di Brescia, Brescia 1982, pp. 98-99; N. D’Acunto, La pastorale nei secoli centrali del medioevo. Vescovi e canonici, in A servizio del Vangelo, pp. 84-88.
} 


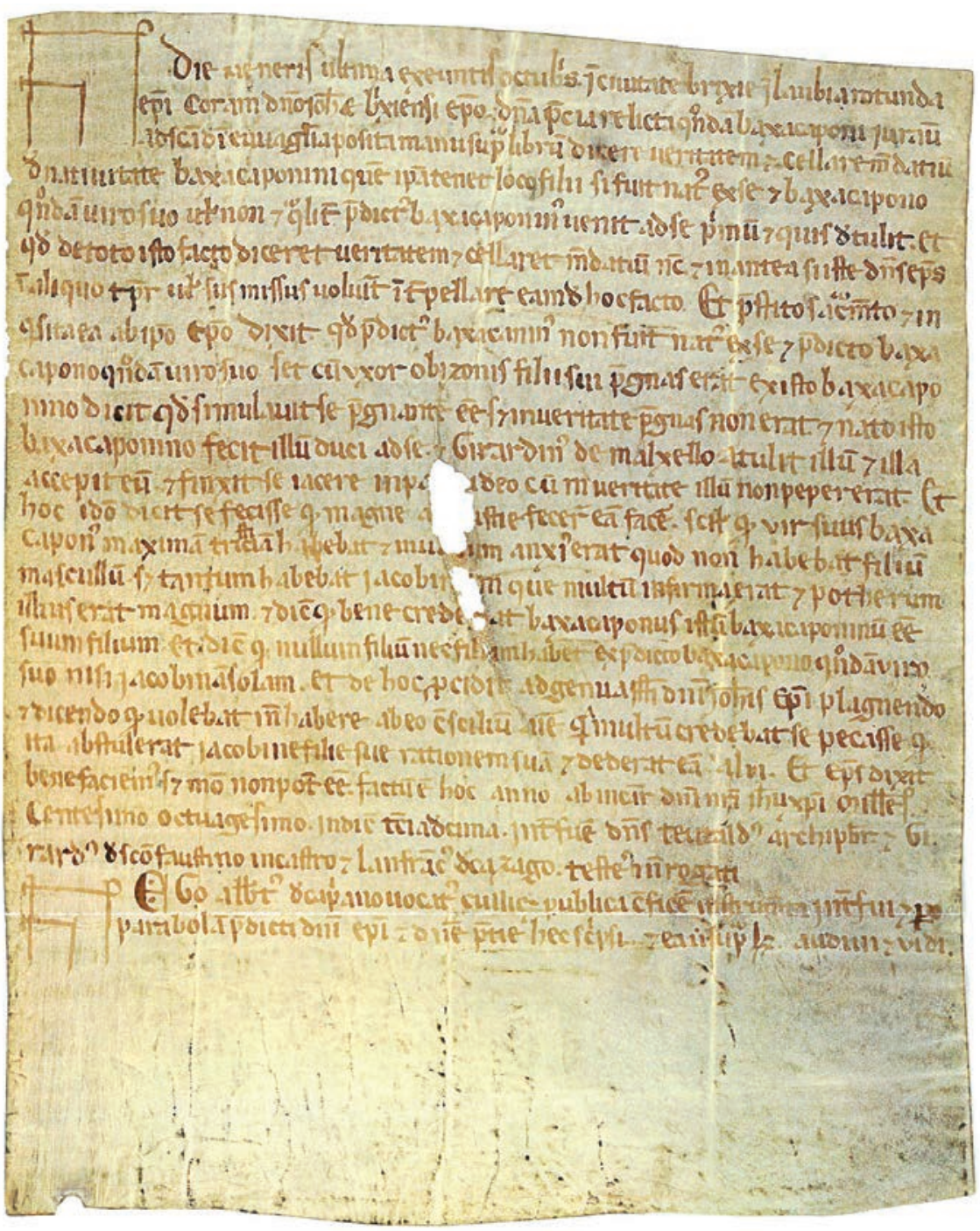

Fig. 1 -Archivio Capitolare di Reggio Emilia, pergamena di denuncia dell'imputata (a. 1180). alla sorella, ritenuta troppo debole per reggere con il necessario vigore il buon nome della casata e il vasto patrimonio - "potherum illius erat magnum» si dice nella deposizione - di cui disponeva ${ }^{4}$. Per questo il dominus de Rodingo, secondo le parole della moglie, era affranto da maximam tristitiam ${ }^{5}$.

La soluzione arrivò quasi improvvisa quando, non sappiamo se calcolata o fortuita (ma dati i protagonisti è più verosimile la prima), essendo rimasta incinta la giovane nuora moglie di Obizo, l'astuta suocera finse di esserlo anche lei e di attendere un figlio simulando la gravidanza all'insaputa del marito (se pregnantem esse) $\mathrm{o}$, più probabilmente, con la sua compiacenza. Al momento del parto, di fronte alla nascita di un nipotino maschio, la nonna mise in atto il suo piano e si fece portare subito il bambino, che mise a giacere accanto a lei come se fosse suo figlio, pur non avendolo generato. La tresca intessuta astutamente dalla donna con i figli, consenziente o meno il nonno, presunto padre, resse fino a quando Baxacaponus rimase in vita e amministrò i suoi beni; divenne però insostenibile non appena questi morì e il giovane Caponinus, affrancatosi dalla potestà dei due tutori legali, mostrò tutta la sua arroganza nella gestione del patrimonio escludendo la sorella e la madre ${ }^{6}$. Il vescovo, l'energico Giovanni da Fiumicello, ascoltò il racconto di Precia che, inginocchiata ai suoi piedi, si rivolse a lui in lacrime, consapevole di aver molto peccato, per ricevere un conscilium anime e ottenere giustizia per la diletta Jacobina, a causa sua ingiustamente privata dell'eredità paterna. Una situazione complicata madre oppressa dal peso di quanto compiuto e soprattutto per le conseguenze a cui aveva portato il suo gesto ${ }^{3}$. Il matrimonio con Baxacaponus - per lei infatti era la seconda volta che convolava a nozze, dopo essere rimasta vedova - era stato allietato dalla nascita di una bambina, Jacobina; suo marito era un miles molto potente, un cavaliere dell'alta aristocrazia capitaneale dei de Rodingo, preoccupato per la mancanza di un figlio maschio, di un erede cioè che potesse subentrare e con troppi interessi di fronte alla quale, sentita la donna, il presule differì la sentenza in attesa di avere tutti gli elementi per emettere un giudizio ${ }^{7}$ (fig. 2).

\section{ARISTOCRAZIA E POTERE}

I protagonisti non sono degli sconosciuti, ma appartenevano all'aristocrazia rurale e feudale della società padana

\footnotetext{
3 «Et de hoc procidit ad genua suprascripti domini Johannis episcopi plangendo et dicendo quod volebat inde habere ab eo conscilium anime, quia multum credebat se peccasse, quod ita abstuleret Jacobine filie sue rationem sua et dederat eam alii» («E riguardo a questo Precia si gettò in ginocchio davanti al suddetto vescovo dominus Giovanni dicendo tra le lacrime che dopo ciò voleva avere da lui consiglio spirituale, perché credeva di aver molto peccato avendo sottratto a sua figlia Jacobina l'eredità e avendola data ad altri», GuYOTJEANNIN, Les lois du sang, p. 780).

${ }_{4}^{4}$ Ibidem, p. 780. Sullo sviluppo dei de Rodingo e i loro vincoli beneficiali con l'episcopato e i grandi cenobi del territorio, cfr. G. ARCHETTI, Signori, capitanei e vassalli a Brescia tra XI e XII secolo, in La vassallità maggiore nel Regno Italico. I capitanei nei secoli XI-XII, Atti del Convegno (Verona, 4-6 novembre 1999), a cura di A. Castagnetti, Roma 2001 (I libri Viella, 27), pp. 161-187.

5 GuYotjeannin, Les lois du sang, pp. 769-770 e 780 per la citazione.

${ }^{6}$ Si vedano ad esempio le parole di Precia riferite nella deposizione di domina Armelina, conversa di Santa Maria di Cure a Montisola, cfr. Ibidem, pp. 785 e 790 per la tutela legale.

7 Ibidem, pp. 779-780. L'esito processuale della vicenda non ci è noto, benché sulla base della denuncia e delle deposizioni giurate, si potrebbe ritenere che sia giunta ad una sentenza di condanna nei confronti della donna e di ripristino del patrimonio in favore di Jacobina o forse di compromesso, ma se si presta fede ad un teste molto autorevole come il dominus Girardo Rangone - che disse come Obizo, padre di Caponino, seppe superare la difficile questione giudiziaria (ivi, p. 789) - si può ipotizzare che i de Salodo abbiano superato positivamente la delicata vertenza. Di Caponino e dei suoi eredi, così come del nonno, esistono vari riferimenti nelle carte monastiche del priorato cluniacense di San Nicolò di Rodengo tra XII e XIII, soprattutto in relazione a quella parte di beni tenuta a Comezzano e a Rudiano per conto dei monaci, cfr. P. GUerRINI, Le più antiche carte del priorato cluniacense di Rodengo (Brescia), «Benedictina», 3 (1949), pp. pp. 77, 81-83, 102-104; L. BEzzI MARTINI, Le pergamene, «I quaderni dell'Abbazia», 3 (1986), pp. 69, 75-76; inoltre, Liber potheris communis civitatis Brixiae, a cura di F. Bettoni Cazzago, L.F. Fè d'Ostiani, in Historiae Patriae monumenta, XIX, Augustae Taurinorum 1899, coll. 659, 988, 998; GuYotjEANnin, Les lois du sang, pp. 769-770, 779-79o passim.
} 


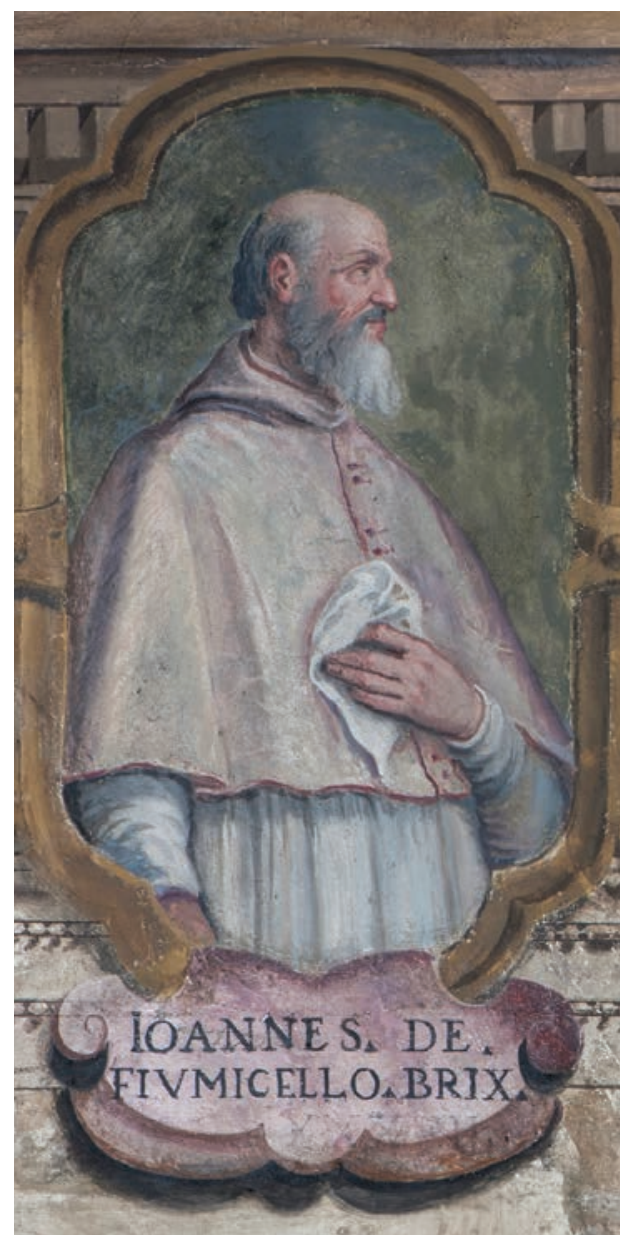

Fig. 2 - Brescia, salone dell'Episcopio, immagine del vescovo Giovanni da Fiumicello.

del tempo. Domina Precia era giunta nel locus di Ome, dove risiedeva in Franciacorta ${ }^{8}$, in seguito al matrimonio con Baxacaponus; in precedenza aveva avuto almeno cinque figli - Urso, Petrus, Casarius, Boxadrus e Obizo -, tutti appartenenti alla famiglia dei de Salodo, una casata nobiliare che aveva il suo punto di forza nei possedimenti posti nell'omonima località gardesana di Salò, legati alla pieve di Santa Maria; in seconde nozze aveva sposato Baxacaponus, esponente del potente lignaggio dei de Rodingo, che compare a Lodi nel 1167 in una riunione della Lega lombarda, come console di Brescia l'anno seguente e apparteneva ai milites di alto lignaggio legati al priorato di Rodengo9. La loro figlia Jacobina era andata in sposa a Milo della schiatta degli Ugoni, la cui famiglia aveva beni in città

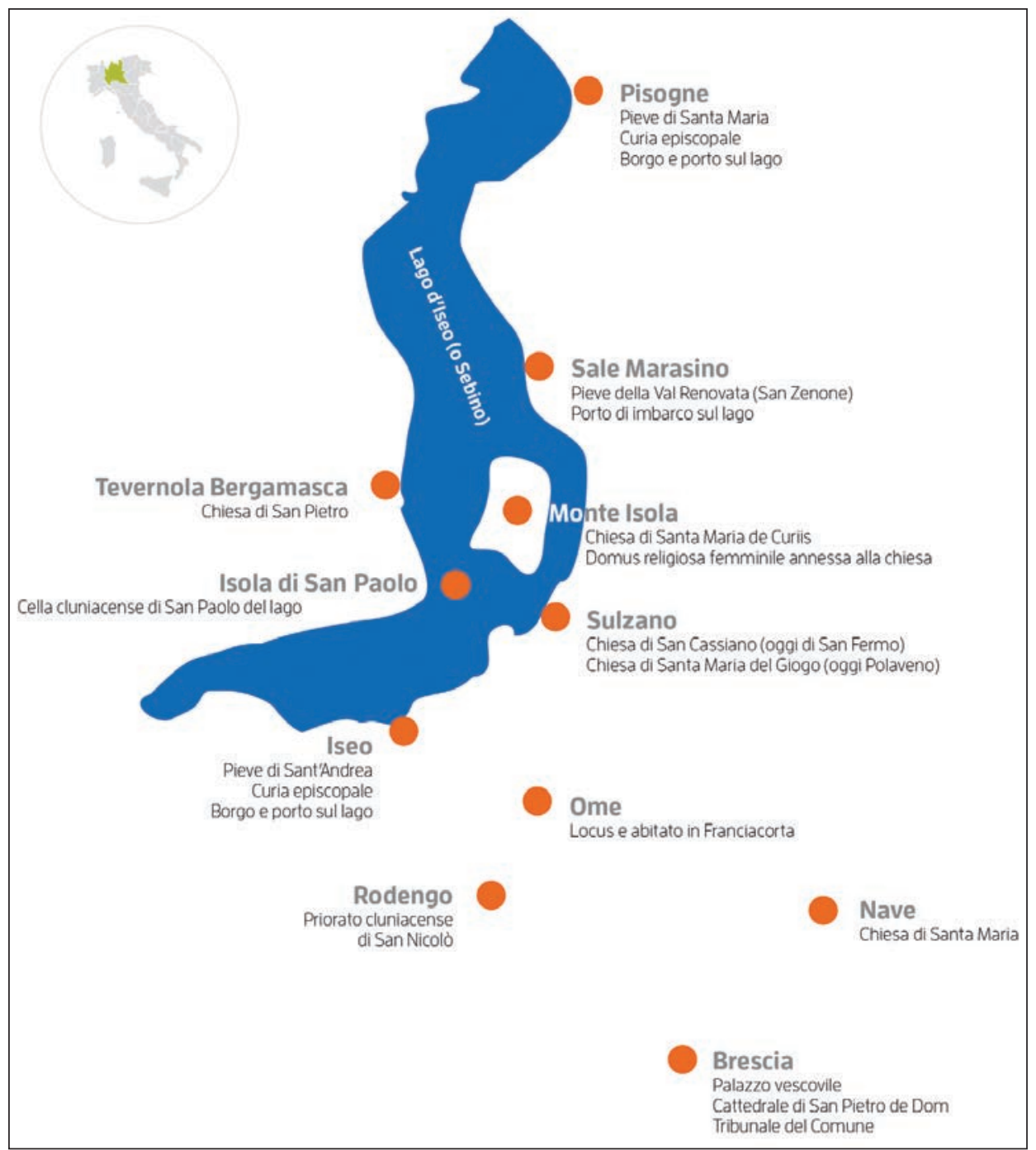

Fig. 3 - La zona del lago d'Iseo e della Franciacorta (Lombardia) interessata dagli spostamenti di domina Precia.

e in varie località del Garda; anch'egli fu console di Brescia nel 1200 e podestà di Ravenna nel $1198^{10}$.

Altri personaggi di prima grandezza, vincolati da rapporti beneficiari con l'episcopato e i centri del potere monastico, sono i domini Madius di Poncarale e Ugutio di Rodengo, tutori del piccono Caponino, Boso Confalonieri, Alberto di Rodengo, Lanfranco di Cazzago, Piardo da Palazzo, Milo dei Griffi, nomi che rimandano tutti alla maggiore nobiltà urbana e del contado, il cui profilo è parte coerente con la storia comunale lombarda del tempo" (fig. 3). Buona parte dei rapporti sociali di cui si dà conto, inoltre, sono

\footnotetext{
8 Posto sulle colline della Franciacorta, cioè nell'area pedemontana tra i fiumi Mella e Oglio ad occidente della città lombarda di Brescia, per il centro di Ome si rimanda al volume La terra di Ome in età medievale, cit.; per il riferimento alla residenza della donna, GuYOTJEANNIN, Les lois du sang, pp. 783-784; ARCHETTI, Abitato e territorio a Ome nel Medioevo, pp. 44-48.

${ }_{9}$ Cfr. A. Bosisio, Il comune, in Storia di Brescia, I, Brescia 1963, p. 628; Statuti bresciani del secolo XIII, a cura di F. Odorici, in Historiae Patriae monumenta, XVI, 2, Augustae Taurinorum 1876, coll. 1582 47; GUERRINI, Le più antiche carte del priorato, pp. 77, 81-83, 102-104; ARCHETTI, "Ad suas manus laborant", p. 91. 10 Statuti bresciani del secolo XIII, col. 1584 47, 51, 53, 73; Liber potheris, coll. 15, 88 (Albertus de Rodingo, a. 1180, 1192); 15, 790 (Pregnaca, a. 1170, 1180); 122, 166, 301, 314 (Ursus de Salodo, a. 1220, 1227); 790 (Madius de Pontecaralo, a. 1170).

"Senza entrare nella prosopografia di tutti i personaggi e degli altri menzionati nel documento, si vedano almeno: Statuti bresciani del secolo XIII, col. 1584 $4^{50-51}$; Storia di Brescia, I, ad indicem; A. BARONIO, Monasterium et populus. Per la storia del contado lombardo: Leno, Brescia 1984 (Monumenta Brixiae historica. Fontes, VIII), ad indicem; GuYotjeannin, Les lois du sang, pp. 768-770; F. Menant, Campagnes lombardes du Moyen Âge. L'économie et la société rurales dans la région de Bergame, de Crémone et de Brescia du Xe au XIII siècle, Rome 1993 (Bibliothèque des Écoles françaises d'Athènes et de Rome, 281), pp. 601-663; ID., Le monastère de S. Giulia et le monde féodal. Premiers éléments d'information et perspectives de recherche, in S. Giulia di Brescia. Archeologia, arte, storia di un monastero regio dai Longobardi al Barbarossa, Atti del convegno (Brescia, 4-5 maggio 199o), a cura di C. Stella e G. Brentegani, Brescia 1992, pp. 119-129; J.-C. MaIRE VIgueUR, Note sugli ufficiali bresciani, in I podestà dell'Italia comunale, a cura di Id., Rome 2000 (Collection de l'École française de Rome, 268), pp. 107-111; ARCHETTI, Signori, capitanei e vassalli, pp. 161-187.
} 


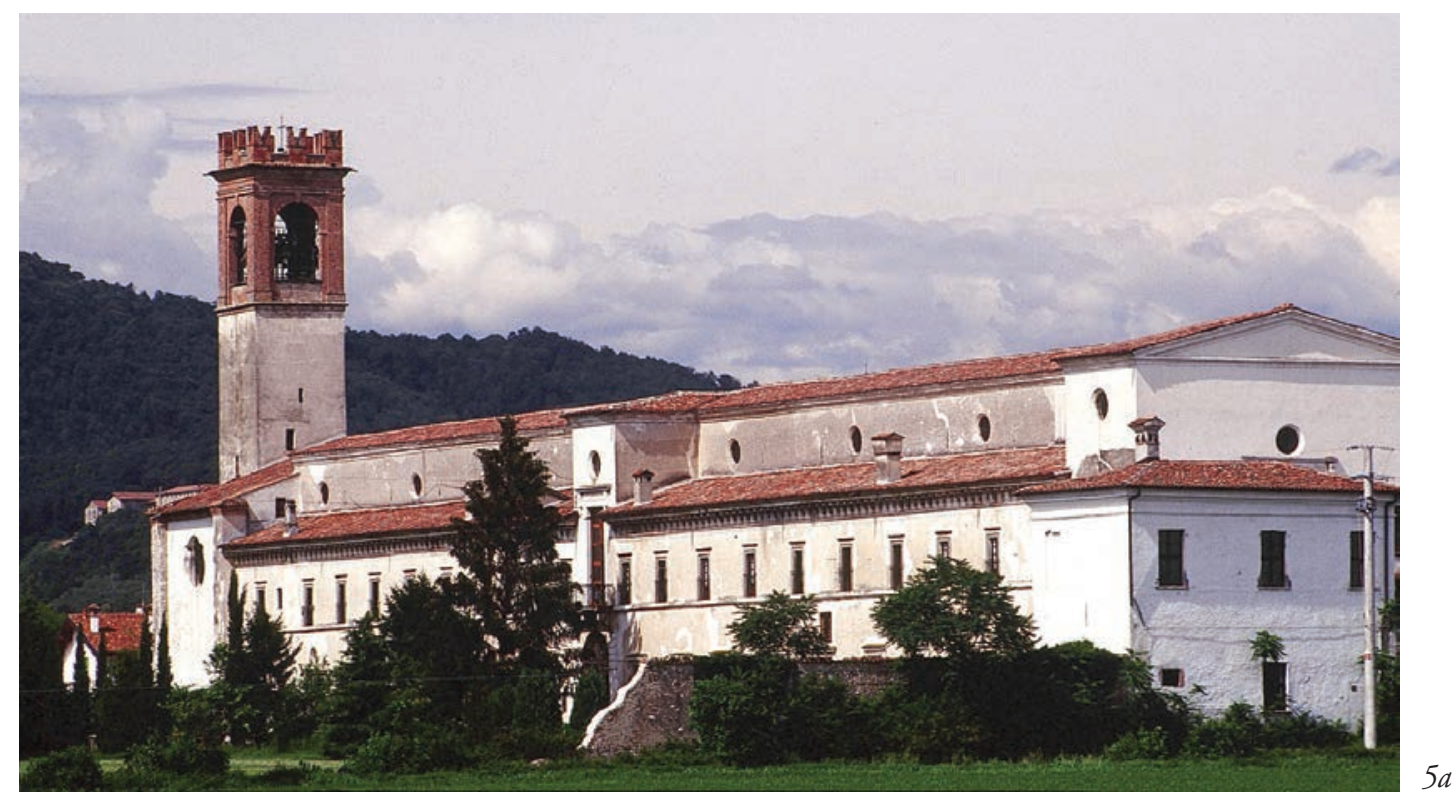

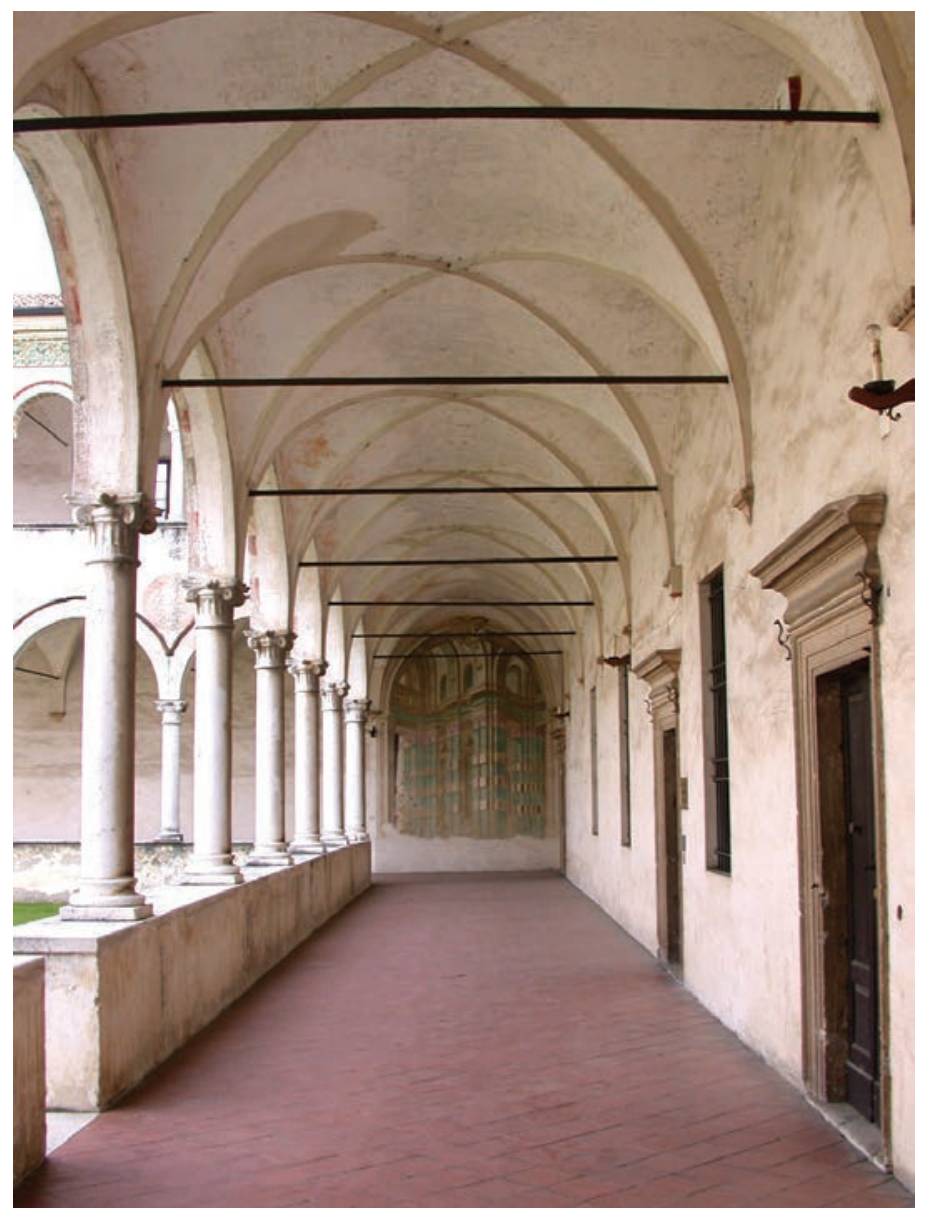

Figg. 5a, 5b-Rodengo (Brescia), monastero olivetano di San Nicolò, eretto sull'antico priorato cluniancense sorto nella seconda metà dell'XI secolo.

imperniati sulle relazioni di potere signorile e dei rapporti feudo-vassallatici, attestati dai termini consilium, auxilium, adjutorium, osculum, honor, investitura, servitium o da espressioni come adiuvare et sustinere, facere sacramentum, suscipere in manibus, dare consilium anime e tenere donnam et majorem honorifice; la chiesa di Santa Maria di Montisola offre a Precia «consiglio e protezione», sollecita il consilium del vescovo e quello dei chierici della pieve di Sale Marasino, competenti per territorio, per fronteggiare le richieste dell'autorità giudiziaria cittadina, i figli obbligano (distringunt) invece la madre ad agire in modo da non essere disonorati, e così via ${ }^{12}$.

Al centro dell'intera questione, che interessò dapprima la giustizia ordinaria e poi quella ecclesiastica, vi era l'eredità o, meglio, il magnum potherum di Baxacaponus di cui erano state escluse la legittima erede Jacobina e poi la madre, come confermano le otto deposizioni giurate raccolte da un notaio e autenticate dal giudice del comune per conto del podestà cittadino $^{13}$ (fig. 4). Dal contesto delle loro testimonianze, appare indubitabile che la paternità del piccolo Caponinus era di Obizo, figlio di Precia, e che dal suo accordo fraudolento con la madre era scaturito l'inganno. Tale in verità forse solo agli occhi del padre, poiché le chiacchiere che si facevano al riguardo erano andate oltre i limiti del locus di Ome, dove Baxacaponus risiedeva e teneva gran parte dei possessi. Alla sua morte, infatti, molti si erano recati a far visita alla salma, vegliata dai monaci cluniacensi tra le mura del priorato di San Nicolò, ma quando i tutori di Caponinus chiesero ai parenti e agli amici del defunto, raccolti nel brolo e nel chiostro del monastero di Rodengo (figg. 5a, 5b), di giurare che avrebbero sostenuto il fanciullo nel prendere possesso del suo pothero, parecchi dei presenti cominciarono a mormorare e a risentirsi sapendo come stavano realmente ed erano andate le cose. Anzi, le deposizione di due autorevoli testi come Milo Griffonis e Pregnaca, consoli di Brescia rispettivamente nel 1190 e nel 1170, che riferiscono il medesimo episodio, sono concordi nel confermare il senso del racconto e la sua veridicità ${ }^{14}$.

\footnotetext{
12 GUYOTJEANNIN, Les lois du sang, pp. 780-79o passim.

${ }_{13}$ Si tratta di Dulcera, conversa di Santa Maria di Cure; di domina Armelina, superiora della medesima comunità di converse della chiesa mariana, figlia del dominus Aldebrando di Mairano; di Vitalino residente presso la chiesa di San Cassiano di Martignago - oggi San Fermo a Sulzano - nel pievato della Val Renovata o di Sale Marasino, sulla sponda orientale del lago d'Iseo; di domina Bontat della chiesa di Santa Maria del Giogo di Sulzano, nel territorio del pievato iseano (oggi Polaveno); di Fiducia de Inzino messo di Precia; del dominus Alberto Pellisdeguerra della città di Modena; infine, dei milites Milo Griffonis e Pregnaca (Ibidem, rispettivamente pp. 781-782, 783-786, 786, 786-787, 787-788, 788-789, 790).

${ }_{14}$ Ibidem, p. 790; Statuti bresciani del secolo XIII, col. 1584 ${ }^{47,51}$; Liber potheris, coll. 15, 790.
} 


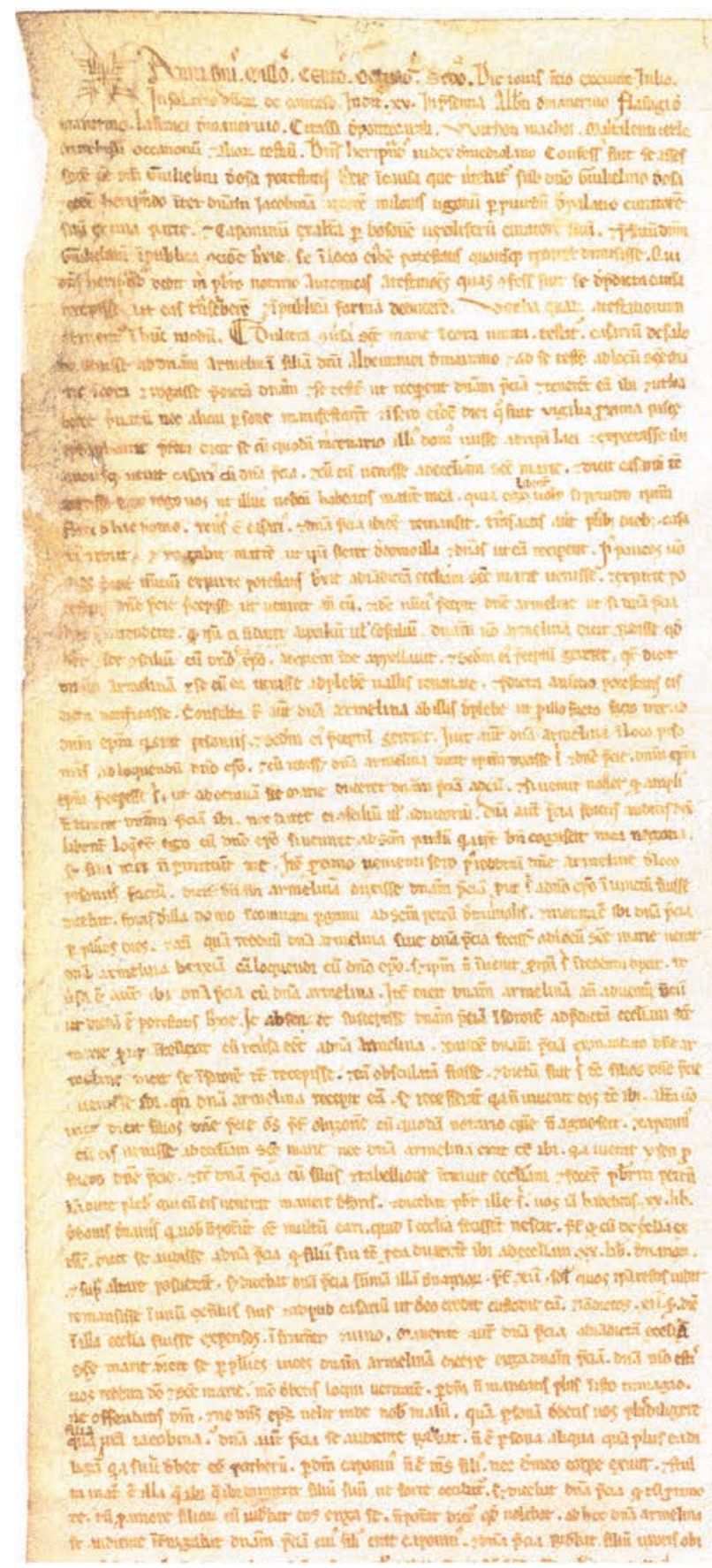

Fig. 4-Archivio Capitolare di Reggio Emilia, pergamena con le deposizioni dei testi chiamati in giudizio (a. 1182).

Milo dei Griff, in particolare, disse che «il giorno nel quale [Baxacaponus] fu sepolto, egli si trovava nel chiostro o nel brolo del monastero dove alcuni dei parenti e degli amici del morto, di cui non ricordava i nomi, [...] giurarono di aiutare e sostenere Caponino nella difesa delle sue ragioni. Fu allora che udì molti uomini rumoreggiare e dire, contro quella gente, che Caponino non era figlio del defunto Baxacaponus. Per questo motivo vide molti astenersi dal pronunciare quel giuramento, cosa che fece anche lui». Aggiunse inoltre di aver udito uno dei figli di Precia, Boxadrus de Salodo, asserire pubblicamente alcuni giorni prima dell'inizio della vertenza nella cattedrale cittadina di San Pietro de Dom: «Per questo santo volto di Pietro, che si trova qui, il giorno in cui Caponino perderà questa causa, non abiterò più a Brescia né in territorio bresciano!»»5. Espressione che manifestava la piena consapevolezza della gravità della vertenza e delle sue prevedibili conseguenze sulla tenuta del patrimonio familiare.

Pregnaca, invece, aggiunse di essere andato anche lui a Rodengo per far visita alla salma, dove nel brolo del cenobio diversi parenti e amici del defunto giurarono in favore dell'eredità di Caponino (de suo pothero); un certo Girardo Guascone però, del quartiere urbano di Portagallia, disse apertamente, interpretando il parere di molti dei presenti alle esequie: «Chiunque vuole pronunciare questo giuramento, può sicuramente farlo ma tanto non ha alcun valore». Gli chiesero perché affermasse ciò, ed egli rispose senza esitazione: «Perché Caponinus non è figlio di Baxacaponus». Che ciò fosse noto trova conferma pure dal fatto che numerosi altri, visto come si mettevano le cose, fecero come lui. E che non vi fossero equivoci sulle generalità del ragazzo lo si apprende dalle parole stesse di Precia che, alla domanda della conversa Dulcera se voleva bene alla figlia, rispose: «Non vi è alcun altra persona che ami più di lei e sua deve essere l'eredità. Per Dio, Caponino non è mio figlio, né è uscito dal mio corpo! $»^{16}$. E proseguiva aggiungendo che era stata lei ad orchestrare tutto con la complicità dei figli.

\section{CAVALCANDO LUNGO LA STRADA PER COSTANTINOPOLI}

Pare che neppure il vero padre, però - e qui si apre un primo squarcio sulla mobilità medievale che va collocato in un momento cruciale della vertenza - fosse particolarmente discreto nel mantenere segreto l'intrigo che aveva visto l'esclusione della sorellastra Jacobina dall'eredità a vantaggio di suo figlio Caponino. Al riguardo è interessante la testimonianza di Alberto Pellisdeguerra, un modenese di elevata condizione sociale al servizio di Girardo Rangone, suo signore ${ }^{17}$. Questi disse che, durante un viaggio a Costantinopoli, avvenuto tra il 118o e il 1181, quando giunsero a Gallipoli, «che è sopra il braccio di San Giorgio», ed erano pronti ad imbarcarsi col loro seguito, sopraggiunse anche Obizo di Salò che chiese si unirsi in societate con loro. Viaggiarono, dunque, insieme fino a Bisanzio dove giunsero di mercoledì, che era la vigilia della festa di Santa Maria Ciriale, cioè il 2 febbraio 1181 giorno della Presentazione al tempio di Gesù, e si trattennero in città per cinque giorni; la festa della Candelora andarono a messa in una chiesa di Costantinopoli, dedicata alla Vergine, e il lunedì della settimana seguente ripartirono facendo tappa dodici miglia a ponente della capitale bizantina nella località chiamata Rezium, l'attuale quartiere turco di Küçükçekmece nella parte europea di Istanbul, per proseguire poi alla volta di Tessalonica e di Durazzo, da dove presumibilmente si imbarcarono per l'Italia. Non conosciamo i particolari del tragitto, né le modalità del loro viaggio che avvenne a cavallo, ma è molto interessante la testimonianza, raccolta attraverso una deposizione testimoniale, di un itinerario per nulla breve e assai impegnativo, anche se non infrequente.

\footnotetext{
${ }_{15}$ GuYotjeannin, Les lois du sang, p. 790 anche pe altre citazioni e per la testimonianza di Pregnaca.

${ }^{16}$ Ibidem, pp. 782,785 .

${ }_{17}$ Ibidem, p. 772 n. 20-21, sui due personaggi e il loro ruolo politico-istituzionale nelle vicende del tempo; per Girardo Rangone - podestà di Modena (1156), legato imperiale per il territorio modenese (1167), console della città (1173) e di nuovo podestà (1179-1180) -, cfr. E.P. VICINI, I podestà di Modena (1156-1796), parte I: 1156-1336, Roma 1913, pp. 33-34.
} 
Ora, durante la strada di rientro verso casa, un giorno del mese di febbraio del 1181, Alberto Pellisdeguerra si trovò a cavalcare a fianco di Obizo di Salò verso l'attuale località albanese di Durazzo (Durrësi) e a chiacchierare con lui del più e del meno (equitando, ludendo et loquendo), cercando di ingannare il tempo nello stesso modo con cui fanno $\mathrm{i}$ viaggiatori odierni. Ad un certo punto il discorso cadde sugli affetti domestici lontani e il vassallo Alberto chiese ad Obizo se fosse sposato, e lui rispose: «Certo, ho una moglie che amo molto, anche più dei figli e dei fratelli». «Perché la amate così tanto?», gli chiese di nuovo e Obizo: «Dovete sapere che c'è stato un tempo in cui sono stato malato e così malconcio che gli uomini mi evitavano. Mia moglie invece non si è allontanata un solo istante dal mio letto, né mi ha mai fatto mancare nulla, anzi mi ha accudito allo stesso modo con cui ogni altro uomo è servito da qualunque donna. Per questo la amo». Alberto riprese: «Signore, avete figli?» e quello: «Ho lasciato a casa due figli..., e un altro, che il Signore mi protegga!». E Alberto di rimando: «Quomodo, unum alium? Come sarebbe a dire un altro?», e Obizo: «Sic est, è proprio così!».

In un crescendo di curiosità il nostro informatore riprese: «Come lo avete avuto, se amate tanto vostra moglie come dite? Lo avete per caso avuto da un'altra donna?», e Obizo: «Non, no!». «Allora in che modo? Per Deum, domine, dicite mihi», replicò Alberto e l'altro rispose: «Signore, dovete sapere che mia madre ha avuto un secondo marito, dal quale però non ha avuto un figlio maschio, ma una sola bambina. Io ho avuto quel figlio da mia moglie e con mia madre ho fatto un patto, in modo tale che lei lo facesse apparire come suo e lo tenesse come figlio. E in effetti può senz'altro tenerlo come il suo bambino dal momento che è mio figlio». Alberto riprese: «Gli volete bene», e Obizo: «Voglio bene a quel bambino tanto quanto a qualsiasi altro dei miei figli. Perché non dovrei volergliene? Dal momento che i suoi fratelli ricevettero magnum honorem et servitium, egli è ricco tanto quanto tutti gli altri $»^{18}$. Come conciliare un simile inganno e la rinuncia alla paternità di un figlio con l'affetto di un genitore per i suoi bambini, se non per la ricchezza? Obizo lo dice chiaramente nella sua ultima risposta: dives est tantum ut ceteri omnes.

Valoroso e saggio, alla fine del XII secolo il cavaliere (miles) doveva essere ricco e stimato, anzi molto ricco, come pure abile nell'arte della parola quasi come nel brandire la spada. Lo ricorda ancora il teste a proposito del medesimo Obizo che viene definito, dal suo dominus Girardo Rangone, in occasione di un momento conviviale del viaggio in uno ospizio della penisola balcanica, "prudente" e "saggio": «Vere prudens et sapiens. Perché se così non fosse - egli osservò -, non sarebbe uscito indenne da così grandi vicende, dalle quali è invece scampato. Infatti ne ha combinata una, che non è stata per niente bella da fare». Alla domanda se ciò fosse dovuto ad un sanguinoso scontro bellico (de facto batallie) a cui era fortunosamente scampato, prese a narrare del complotto ordito con la madre per lucrare l'eredità in modo disonesto ai danni di Jacobina, per cui «diede uno dei suoi figli a sua madre, la quale lo fece suo e lo tenne come se fosse suo figlio, poiché era la moglie di un cavaliere ricco che non aveva avuto da lei un erede maschio» ${ }^{19}$.

Lo dimostra altresì l'uso costante della terminologia feudale applicata alle diverse sfere della vita sociale e l'impiego di varie scappatoie giuridiche pur di garantirsi il successo nell'attribuzione del patrimonio di Baxacaponus. La confessione di Precia tuttavia, dopo la denuncia presentata da Jacobina e dal marito Milo al tribunale di Brescia, se aveva aperto alla giustizia ecclesiastica la possibilità di fare il suo corso e forse alleggerito la sua coscienza, giungeva comunque tardivamente e inquinata di nuovi interessi. Essa innescò subito l'azione difensiva dei figli che a questo punto rischiavano seriamente di perdere insieme ai beni usurpati anche l'onore, come indicano le parole quasi di imprecazione di uno di loro, Boxadrus de Salodo, pronunciate nella cattedrale cittadina: «Cosa pensano di fare? Per Dio, il giorno in cui Caponino perderà la causa, io e i miei fratelli non abiteremo più a Brescia! ${{ }^{20}}^{20}$ Non va del resto trascurato il fatto che, se all'origine dell'inganno c'era stato il primogenito Obizo in combutta con la madre, nel momento in cui viene messo in pericolo l'onore della famiglia, tutti i suoi membri si uniscono per difendere interessi e reputazione comuni, come dei «consorti senza consorteria» per usare una felice espressione di Gabrielle Rossetti, da cui emerge anche lo spaccato di un sistema familiare in rapida trasformazione verso il patrilignaggio, dove il riconoscimento delle prerogative ereditarie al primogenito diventava il sistema principale per la conservazione - talvolta in forme consortili - del patrimonio familiare ${ }^{21}$.

\section{NELL'ISOLAMENTO RELIGIOSO DI MONTISOLA}

Madre e figli sembrano uniti non solo dai vincoli di sangue ma anche dalla stessa spregiudicatezza e, all'abile determinazione di Precia nell'assicurare ai de Salodo il patrimonio del marito, si contrappone il violento comportamento dei figli quando apprendono della confessione fatta al presule, gesto che aveva portato alla luce, mettendola a nudo, la loro frode. Persuasione, affetto filiale, soprusi, sentimenti religiosi e strategia processuale si mescolano nelle loro decisioni successive alla denuncia della donna. Dopo il disorientamento iniziale, essi si recano dalla madre «che abitava nel luogo di Ome», come si legge nella testimonianza della conversa Armelina della famiglia dei de Mairano, la chiudono in casa, le riducono i viveri e le fanno il lavaggio del cervello riuscendo infine a strapparle una formale ritrattazione. Alla domanda perché si fosse comportata in un modo così contraddittorio, sporgendo denuncia e poi ritirandola, Precia rispose: «Non ho potuto fare altro, perché i miei figli con i loro e con i miei parenti si sono riuniti in casa mia svergognandomi e rimproverandomi aspramente,

\footnotetext{
${ }_{18}$ Guyotjeannin, Les lois du sang, pp. 788-789.

19 Ibidem, p. 789. Su questa concezione del miles utili le osservazioni di C. Violante, Motivi e caratteri della "Cronica” di Salimbene, «Annali della Scuola Normale Superiore di Pisa. Lettere, storia e filosofia», ser. II, 21-22 (1953), pp. 108-154.

2o Guyotjeannin, Les lois du sang, p. 790, a riferirle era il teste Pregnaca.

${ }^{21}$ G. Rossetti, Histoire familiale et structures sociales et politiques à Pise aux XI' et XII' siècles, in Famille et parenté dans l'Occident médieval, Actes du colloque de Paris (6-8 juin 1974), Rome-Paris 1977 (Collection de l'École française de Rome, 30), p. 175; GUYOTJEANNIN, Les lois du sang, p. 777; inoltre, C. VIOLANTE, Alcune caratteristiche delle strutture familiari in Lombardia, Emilia e Toscana durante i secoli IX-XII, in Famiglia e parentela nell'Italia medievale, a cura di G. Duby e J. Le Goff, Bologna 1977, pp. 19-57; sull'evoluzione del concetto di famiglia e della trasmissione ereditaria patrilineare, con riferimento all'area in questione, G. ARchetTi, Introduzione: famiglie e territorio nel Medioevo fra storia e storiografia, in Famiglie di Franciacorta nel medioevo, Atti della VI Biennale di Franciacorta (Coccaglio, Villa Calini, 25 settembre 1999), a cura di G. Archetti, Brescia 2000, pp. 9-40.
} 


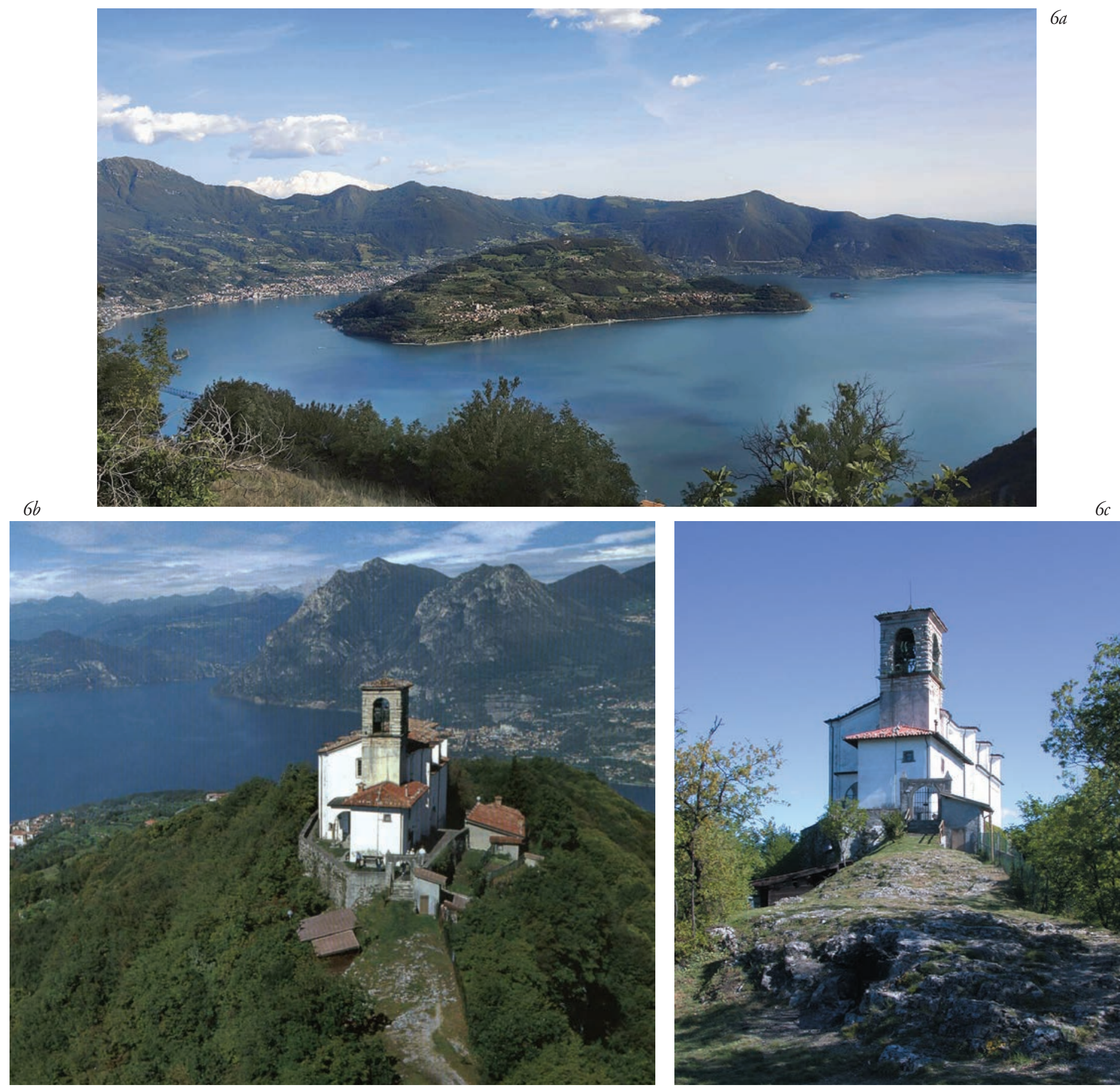

Figg. 6a, 6b, 6c-Montisola (Brescia, lago d'Iseo), santuario della Madonna della Ceriola, edificato sul luogo della chiesa di Santa Maria de Curiis sulla cima del monte.

poi mi hanno minacciata. Non potevo sfuggire a ciò che avevo fatto $»^{22}$. In effetti, il suo atteggiamento arrendevole nei loro confronti e incapace di autonomia mostrava tutta la sua debolezza ogni qualvolta li incontrava ${ }^{23}$.

Di fronte ad un tale comportamento si cercò di verificare la buona fede dell'imputata e il suo equilibrio psicofisico. Alla domanda se Precia fosse pazza (mente captam), stupida (minoris sensus solito) o spesso ubriaca (captam a vino), domina Armelina non dubitò ad affermare che la donna aveva detto certo molte cose inopportune, ma non era né pazza, né stupida, né dedita al vino; aggiunse inoltre che non era neppure amalvegatam, cioè sotto l'influsso di qualche maleficio o sortilegio demoniaco ${ }^{24}$. Ciò non basta: l'apertura della causa processuale prima della sua confessione, intentata davanti al tribunale del podestà di Brescia, il milanese Guglielmo de Osa ${ }^{25}$, da Jacobina contro Caponinus, che avrebbe potuto portarli presto alla rovina, li costringe a giocare d'astuzia per guadagnare tempo. I da Salò prendono la madre e la "persuadono" con ogni mezzo, costringendola alla fine ad indossare l'abito religioso nella chiesa di Santa Maria de Cora, cioè a Santa Maria di Cure sopra Montisola nel lago d'Iseo ${ }^{26}$ (figg. 6a, 6b, 6c), dove la donna viene accolta

${ }^{22}$ GuYOTJEANnin, Les lois du sang, pp. 784 e 783, 781.

23 Ibidem, pp. 785-786; ArchetTI, Abitato e territorio a Ome nel Medioevo, pp. 44 sgg.

24 GuYotjeannin, Les lois du sang, p. 785.

${ }_{25}$ Statuti bresciani del secolo XIII, col. 158450; Bosisio, Il comune, p. 633; L. Armstrong, Brescia, in Medieval Italy an Encyclopedia, by C. Kleinhenz, London 2004, p. 156. 


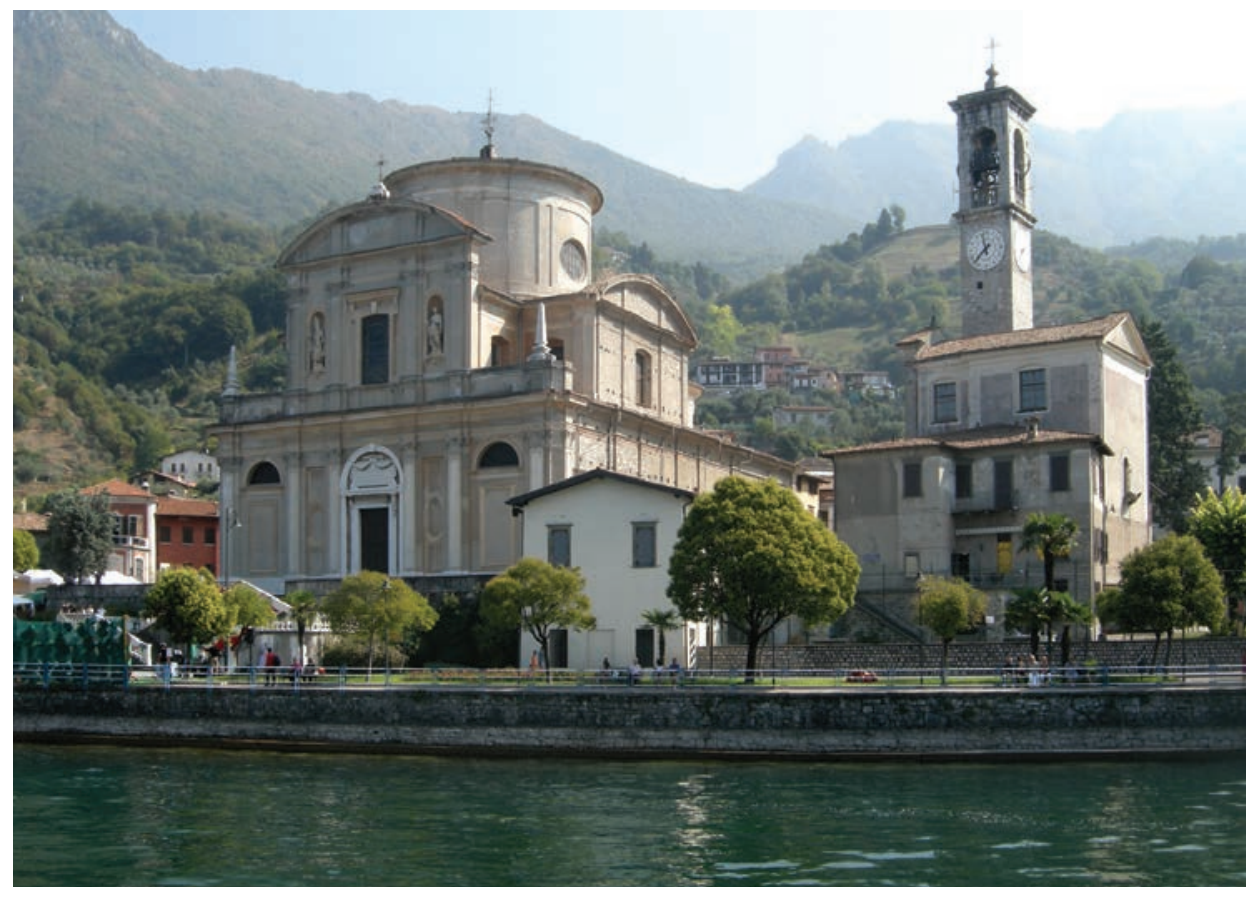

È in questo contesto, infatti, che potevano svilupparsi esperienze di vita religiosa particolari o di tipo quasi eremitico, autorizzate dall'ordinario locale o legate al clero pievano e alla responsabilità dell'arciprete. Per limitarci al territorio bresciano ne abbiamo un esempio a San Vigilio di Caruca, nei pressi dell'abitato di Padernello, nell'attuale comune di Borgo San Giacomo ${ }^{28}$. Annesso a questo edificio sacro campestre risiedeva un gruppo di donne facenti vita comune, soggette all'autorità del pievano; non si trattava, però, né di una comunità monastica nel senso stretto, né di una di tipo canonicale secondo la regola agostiniana. Al contrario, rientrava in quella galassia di istituzioni locali, difficilmente inquadrabili nelle forme religiose canonicamente regolari, sbocciate nella feconda stagione seguita alla riforma della Chiesa. Essa dipendeva dall'ini-

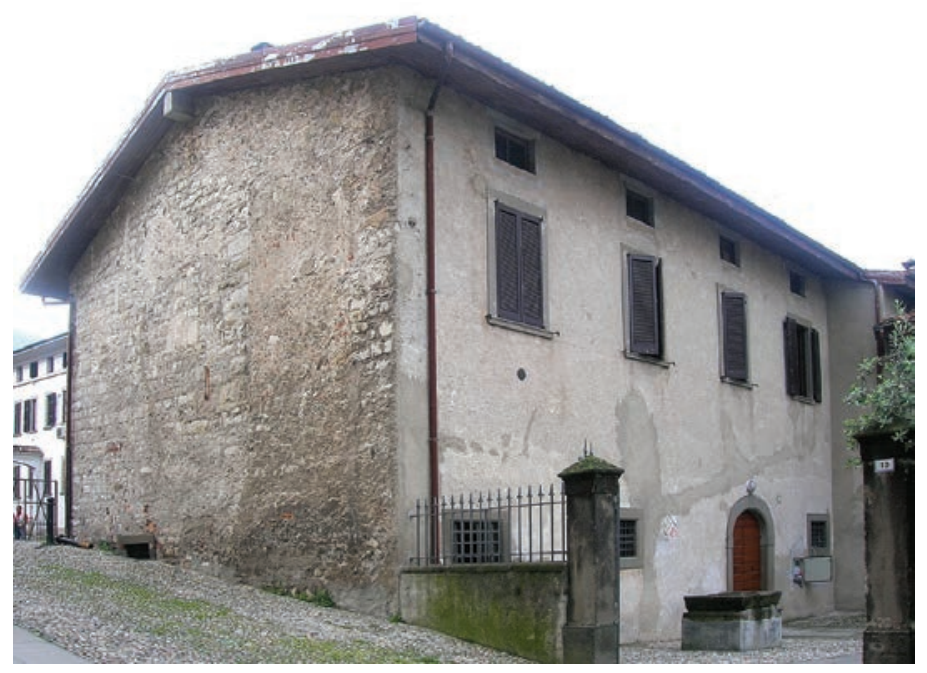

Figg. 7a, 7b-Sale Marasino (Brescia), chiesa di San Zenone eretta sul luogo dell'antica pieve della Val Renovata e particolare della muratura romanica della canonica.

come conversa con l'abbraccio fraterno e il bacio di pace dalla superiora della domus, decisione canonica sancita dai chierici della pieve di San Zenone di Sale Marasino, in Val Renovata (figg. 7a, 7b), sotto la cui giurisdizione ecclesiastica ricadeva la fondazione religiosa ${ }^{27}$. Si tratta di un dato documentario che non trova altri riscontri d'archivio, altresì significativo per il periodo e per lo spiraglio conoscitivo che apre sulle forme femminili di consacrazione religiosa diffuse a livello rurale. ziativa del rettore di Oriano, Teutaldo, che ne consolidò la struttura istituzionale concedendo a Ficia, la superiora, quanto la chiesa di San Vigilio doveva alla pieve dei diritti ecclesiastici (decime, primizie e altre oblazioni) dietro il canone ricognitivo di una libbra di cera. La congregatio mulierum di Padernello, guidata da una prelata, prestava in questo modo il suo servizio di preghiera e di assistenza liturgica presso la ecclesia di San Vigilio.

La regolarizzazione della comunità avvenne ad opera del vescovo Manfredo nel 1146, che la pose sotto la sua protezione e la inquadrò nell'ambito benedettino, consentendo a quelle mulieres di eleggersi la badessa, subordinata alla sua consacrazione, e di scegliersi un presbitero per il servizio sacramentale. Su di lui sarebbe gravato anche l'onere della cura animarum, pur nell'ambito della giurisdizione pievana, dei fedeli legati alla chiesa, ai quali era permesso farsi seppellire presso di essa; un impegno pastorale che poteva contare sulla conferma delle rendite decimali già concesse in precedenza dall'arciprete Teutaldo. Prerogative in parte simili furono attribuite nel 1153 da Anastasio IV alle monache di San Pietro di Fiumicello ${ }^{29}$; il pontefice in effetti, presa la comunità sotto la sua protezione, la esentò dal pagamento delle decime concedendo al cenobio i diritti di sepoltura - nella salvaguardia delle competenze ecclesiastiche della matrice - e la facoltà di eleggersi la badessa.

Un'esperienza analoga è quella attestata sul monte Conche, nel pievato di Nave (fig. 8), ad opera di san Costanzo con la fondazione della chiesa di Santa Maria, per la cura della quale fu istituita una piccola comunità di donne, la

\footnotetext{
${ }^{26}$ Su Santa Maria della Ceriola o di Cure a Montisola del lago d'Iseo e la comunità femminile a servizio di quella chiesa nel XII secolo, v. ARCHETTI, Singulariter in heremo vivere, pp. 113-115; inoltre, P. GUERRINI, Per la storia dell'organizzazione ecclesiastica della diocesi di Brescia nel medio-Evo. Il catalogo capitolare delle chiese e dei benefici compilati nell'anno 1410, «Brixia sacra», XV, 5 (1924), p. 129; Visita apostolica e decreti di Carlo Borromeo alla diocesi di Brescia, III. Sebino, Franciacorta e Bassa occidentale, a cura di A. Turchini, G. Donni, G. Archetti, Brescia 2004 (Brixia sacra, IX, 2), pp. 66, 68.

${ }_{27}$ Riguardo alla chiesa matrice della Val Renovata, posta sulla sponda orientale del Sebino, v. GuERRINI, Per la storia dell'organizzazione ecclesiastica, p. 128; ID., La pieve di Sale Marasino, «Memorie storiche della diocesi di Brescia», 3 (1932), pp. 3-17; Visita apostolica e decreti, III, pp. 41-49; Storia ed arte nella chiesa di San Zenone a Sale Marasino, a cura di F. Frisoni e A. Burlotti, Marone (Bs) 2007, pp. 17-25.

${ }^{28}$ Le carte del monastero dei Santi Cosma e Damiano (Brescia) 1127-1275, a cura di P. Merati, Brescia 2005 (Codice diplomatico bresciano, 2), i docc. 1-4, 9, 105, 152-154, 162 per questa fondazione rurale; le carte erano già state messe in luce da P. GuerRINI, Il monastero di S. Maria di Manerbio e il priorato di S. Maria di Fontana coperta nel territorio bresciano, «Benedictina», XI (1957), pp. 15-30, mentre per la chiesa di San Vigilio, v. A. FAPPANI, S. Vigilio di Padernello, «Brixia sacra. Memorie storiche della diocesi di Brescia», n.s., VIII, 1-2 (1973), pp. 29-35; ArchETTI, Evangelium nuntiare, pp. 293 -294.

29 Le carte del monastero, pp. 13-16, doc. 6; doc. 4, per il provvedimento del vescovo Manfredo.
} 


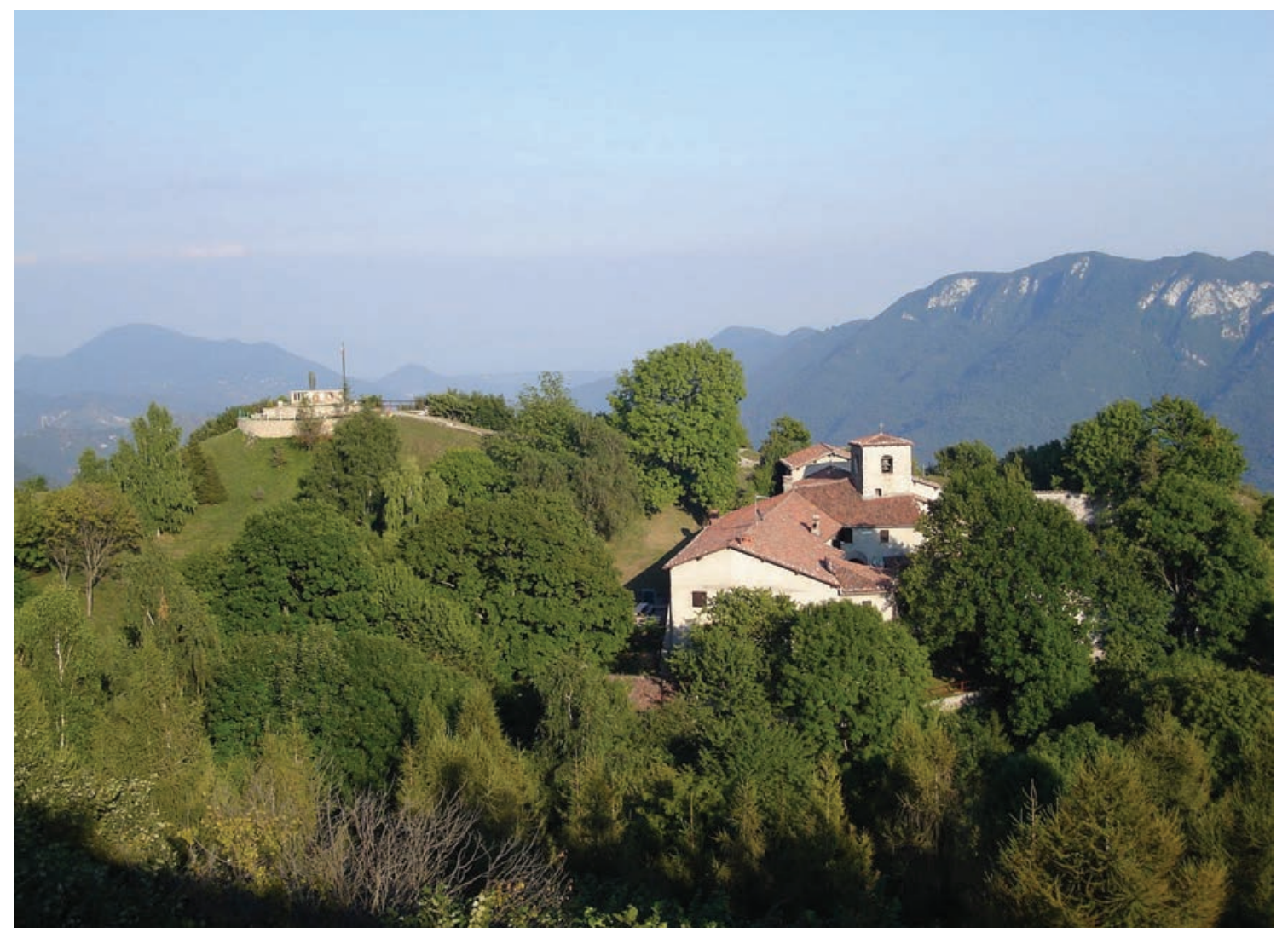

Fig. 8-Nave (Brescia), chiesa di Santa Maria di Conche fondata da san Costanzo.

cui scelta religiosa venne approvata dal vescovo Arimanno intorno al 1110, che consacrò l'edificio sacro, trasformandolo in un presidio della riforma papale; tale esperienza fu poi confermata da numerosi privilegi della Sede apostolica, a cui l'eremo era direttamente soggetto ${ }^{30}$. Nel 1158 la superiora della domus di Conche ottenne dal delegato papale un documento nel quale si confermava la protezione apostolica, si concedeva la facoltà alle sorores di eleggersi una responsabile (prelata), a cui spettava la piena amministrazione dei beni, e veniva fissato un canone ricognitivo in denaro per la dipendenza dalla Santa Sede ${ }^{31}$. Il 27 gennaio 1200 Innocenzo III assicurò ad Agnese, magistra delle monache di Conche il favore pontificio, poi ribadito in un documento del $1213^{32}$.
I termini usati in questi provvedimenti però - domus per indicare la comunità, prelata e magistra per la superiora, sorores per le consorelle - non rimandano ad una realtà monastica, ma ad una comunità di tipo canonicale vivente in povertà e castità secondo regole proprie ispirate a quelle agostiniane ${ }^{33}$.

Ancora, l'esistenza di gruppi di donne e uomini a servizio delle chiese rurali, è attestata a San Pietro di Liano, come si apprende da una missiva di Urbano III del 1186 o 1187, in cui il pontefice, ponendo termine ad un'accesa vertenza tra il vescovo di Brescia Giovanni II e la pieve di Salò, riconosceva al presule la facoltà di insediarvi conversi o converse «vel alias personas» ${ }^{34}$, mentre un gruppo di mulieres curava la

\footnotetext{
$3^{\circ}$ C. Doneda, Notizie di S. Costanzo e Memorie istoriche del monastero di Santa Catterina di Brescia, Brescia 1756; G. GuAdAGnINI, Memorie de' Santi confessori di Cristo Costanzo ed Obizio di Niardo, Brescia 1791 (rist. anast., Malegno [Bs] 1998); G. BrunATI, Vita o gesta di santi bresciani, II, Brescia 1856, pp. 3-9; Acta Sanctorum, Februarii, II, Parisiis et Romae 1864, p. 605: «De S. Constantio apud Brixiam in Italia»; P.F. KeHR, Regesta Pontificum Romanorum, in Italia Pontificia, VI, 1, Berolini 1913, pp. 339-340; R. VAN DOREN, s.v., Constantius, in Dictionnaire d'histoire et de géographie ecclésiastiques, XIII, Paris 1956, col. 769; A. FAPPANI, s.v., Costanzo, in Bibliotheca sanctorum, IV, Roma 1964, col. 266; ARCHETTI, Singulariter in heremo vivere, pp. 116-126; ID., "Terra circondata da monti". Nave e il suo territorio in età medievale, in Nave nella storia dalle origini alla prima età napoleonica, a cura di C. Sabatti e A. Minessi, Brescia 2011, pp. 6o-67. Sulla politica papale di Arimanno a questo riguardo, cfr. ARCHETTI, Evangelium nuntiare, p. 284; F. STROPPA, Memoria della riforma: Arimanno a Brescia, in Medioevo: immagine e memoria, Atti del convegno internazionale di studi (Parma, 23-28 settembre 2008), a cura di A.C. Quintavalle, Milano 2009, pp. 396-407; EAD., Le rotonde, le tombe e le reliquie nella diocesi di Brescia, in Medioevo: le officine, Atti del convegno internazionale di studi (Parma, 22-27 settembre 2009), a cura di A.C. Quintavalle, Milano 2010, p. 411.

${ }^{31}$ Doneda, Notizie di S. Costanzo, p. 43; KeHr, Regesta Pontificum Romanorum, p. 339.

32 Doneda, Notizie di S. Costanzo, pp. 45-46; KeHr, Regesta Pontificum Romanorum, p. 340.

33 La comunità sopravvisse fino al primo terzo del XIII secolo quando nell'ottobre 1235 il legato papale affidò all'arciprete della cattedrale l'inquisitio nei confronti delle sorores di Conche, da cui emerse che a causa della pericolosità del momento non si doveva "permettere che in quella solitudine montana abitassero donne da sole»; di qui la decisione nel febbraio 1236 di annettere la comunità femminile con quella degli umiliati di San Luca di Brescia. DoNEDA, Notizie di S. Costanzo, pp. 47-48; Les registres de Grégoire IX, recueil des bulles de ce pape publiées ou analysées d'après les manuscrits originaux du Vaticain par L. Auvray, II, Paris 1907, col. 406, nr. 3184 (Terni, 12 giugno 1236); ARCHETTI, Terra circondata da monti, pp. 6o-63.

34 KeHr, Regesta Pontificum Romanorum, p. 352; P. Guerrini, La prepositura di San Pietro di Liano a Volciano, «Memorie storiche della diocesi di Brescia», 1 (1930), pp. 33-63; G. ARChetTI, Berardo Maggi vescovo e signore di Brescia. Studi sulle istituzioni ecclesiastiche e sociali della Lombardia orientale tra XIII e XIV secolo, Brescia 1994 (Fondamenta. Fonti e studi di storia bresciana, 2), pp. 142-143; M. TREBESCHI, La serie "culto divino" nell'archivio antico del comune di Salò, «Brixia sacra. Memorie storiche della diocesi di Brescia», III serie, IV, 3 (1999), pp. 176-177; R. Salvarani, Garda romanico. Pievi, istituzioni, territorio, Milano 2004, p. 149; ARCHETTI, Pievi e monasteri in età romanica, pp. 175-176.
} 


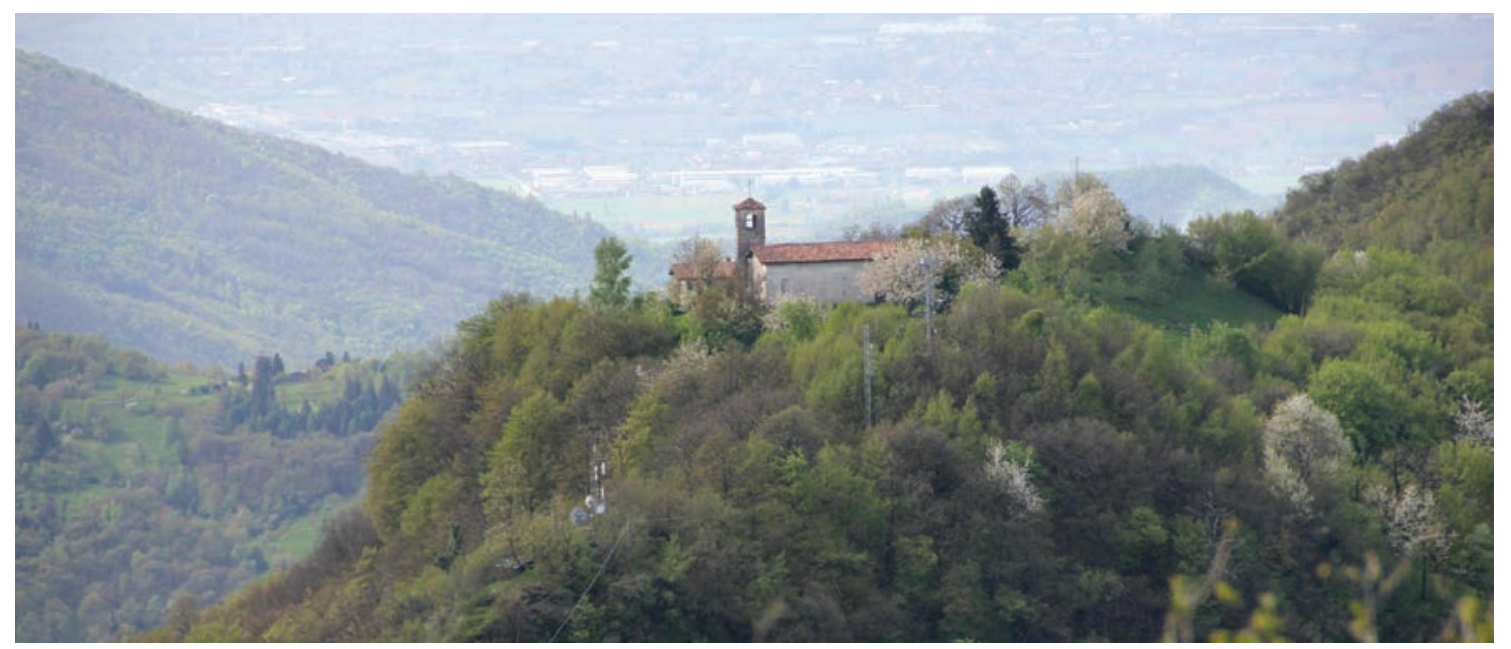

Fig. 9- Sulzano (Brescia), chiesa di Santa Maria del Giogo (oggi nel comune di Polaveno).
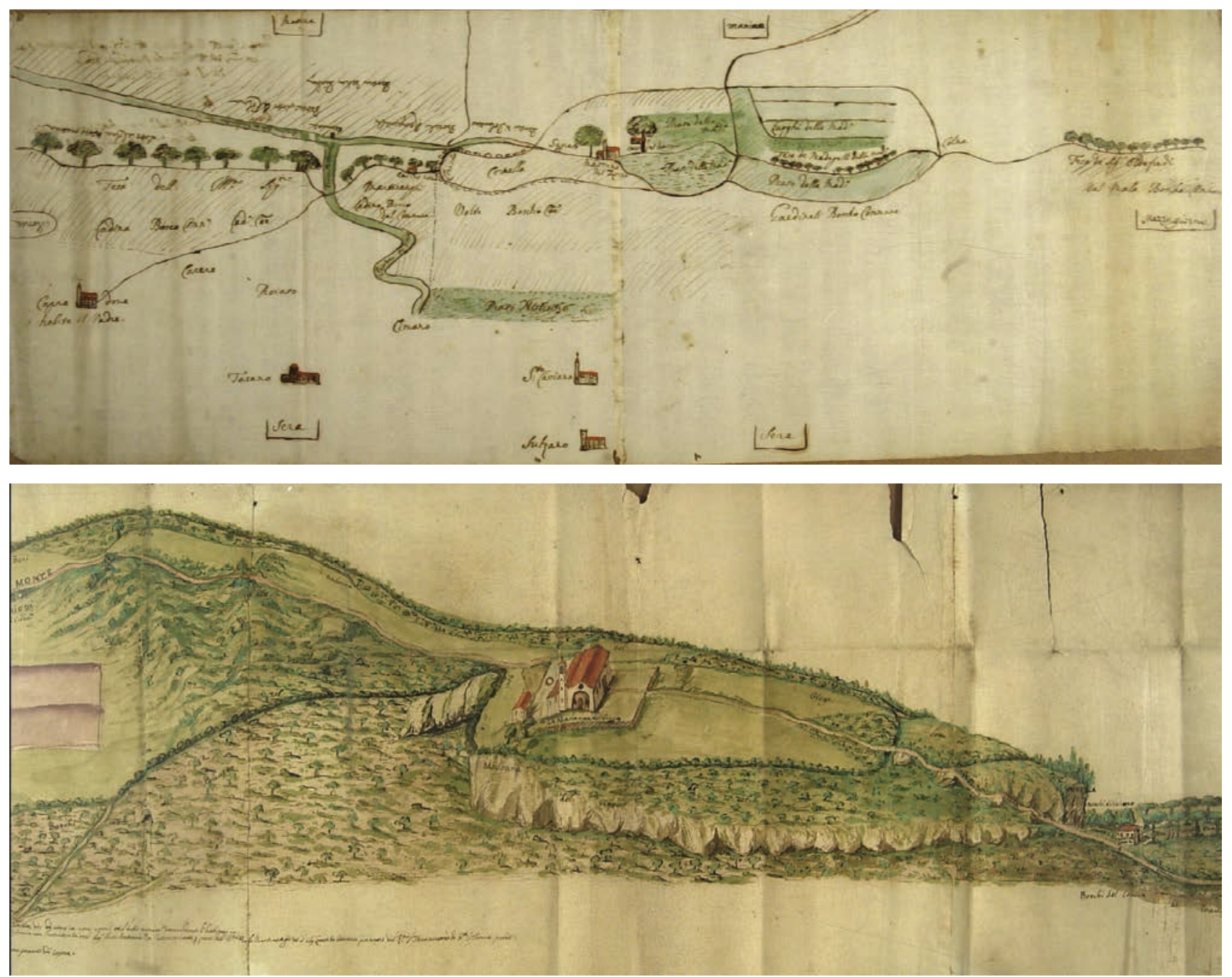

Figg. 10a, 10b-Archivio di Stato di Brescia, chiesa di Santa Maria del Giogo con i possedimenti ed eremo di San Mauro sopra Sulzano in un cabreo tardo seicentesco.

chiesa di Santa Maria del Giogo (in Zuvo) sul monte Capra a Sulzano (fig. 9), nel distretto plebano della Val Renovata, come si apprende dalla deposizione di un'altra teste del nostro processo, domina Bontat ${ }^{35}$. A lei era ricorsa Precia per alcuni servizi personali, la quale risiedeva presso quella chiesa insieme a uomini e donne che conducevano vita separata, se sono riferibili ad essa la monaca Flora e il converso
Ugo alle dipendenze della medesima Bontat. Di questa comunità non sappiamo nulla di più, ma qui il ricordo della vita solitaria alla fine del medioevo era mantenuto vivo dai monaci di Sant'Eufemia, che la officiavano nel periodo estivo occupando anche il vicino eremo di San Mauro in località Capra sopra Sulzano e garantendo una presenza religiosa documentata ancora in età moderna ${ }^{36}$ (figg. 10a, 10b).

35 GUYOTJEANNIN, Les lois du sang, pp. 786, 788, 771. Su questo edificio di culto, cfr. GUERRINI, Per la storia dell'organizzazione ecclesiastica, p. 128; Visita apostolica e decreti, III, pp. 76, 81-82; Polaveno nella storia e nell'arte, a cura di C. Sabatti, Brescia 2003, pp. 24, 28-30, 36, 53, 63-64, 75, 123.

${ }^{36}$ ARchetTI, Singulariter in heremo vivere, pp. 150-154. 

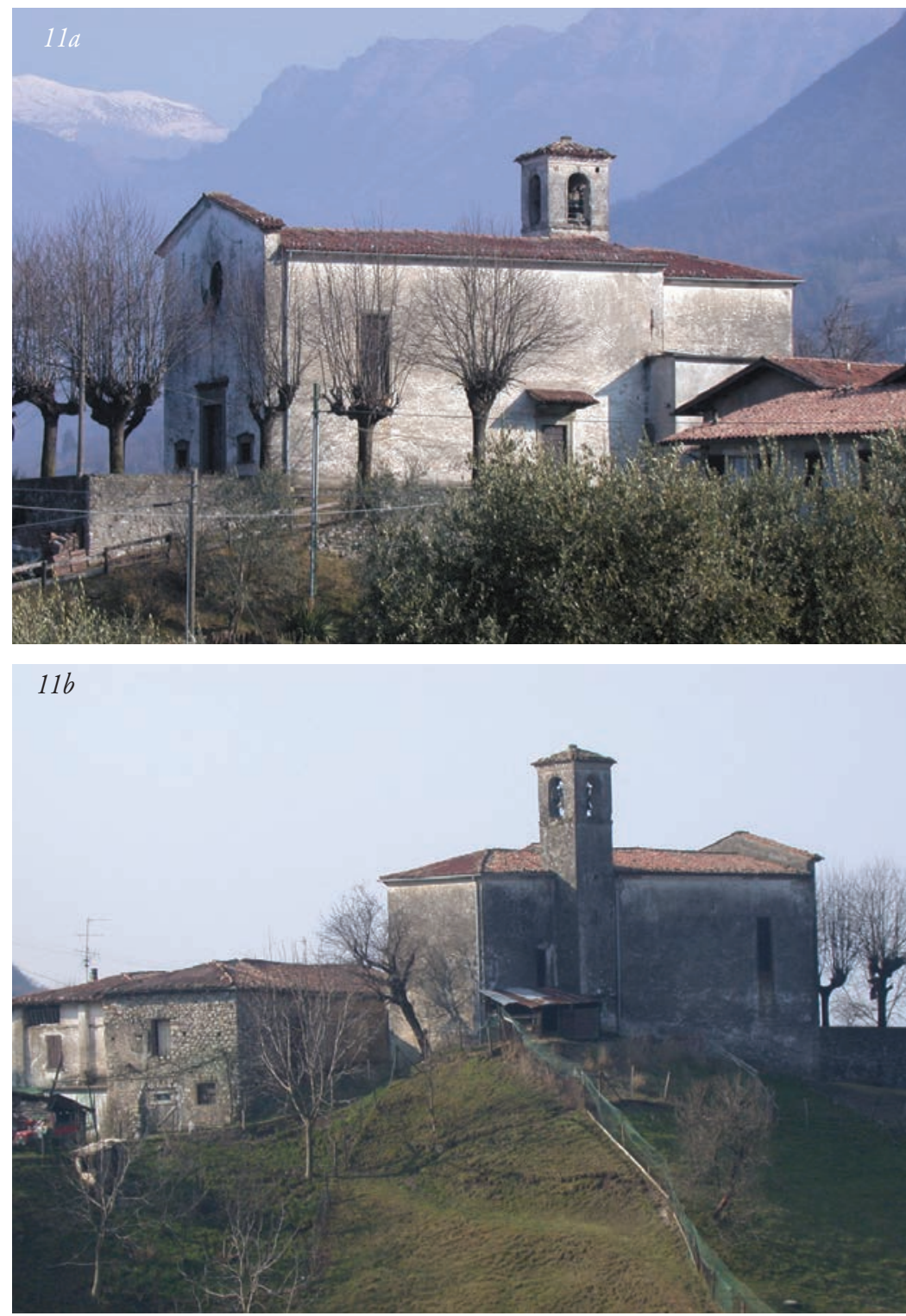

Figg. 11a, 11b, 11c-Sulzano (Brescia), chiesa di San Fermo, già dedicata a san Cassiano, con resti di murature romaniche.

Ma è nell'internamento di Precia a Cure, sulla sommità di Montisola, che prende luce un'esperienza femminile di "eremitismo cenobitico"37, per usare un'efficace espressione di Cécile Caby, altrimenti del tutto sconosciuta. Non è noto da quanto tempo fosse attiva la comunità, anche se è verosimile pensarla sulla spinta della riforma, come farebbero ipotizzare la sua struttura canonicale e la dipendenza dalla pieve di Sale Marasino. La chiesa di Santa Maria de Curiis era affiancata da una domus, della quale sono ricordati il dormitorio e una stanza riscaldata «in qua ignis est», dove vivevano col nome di converse e sorores donne di estrazione

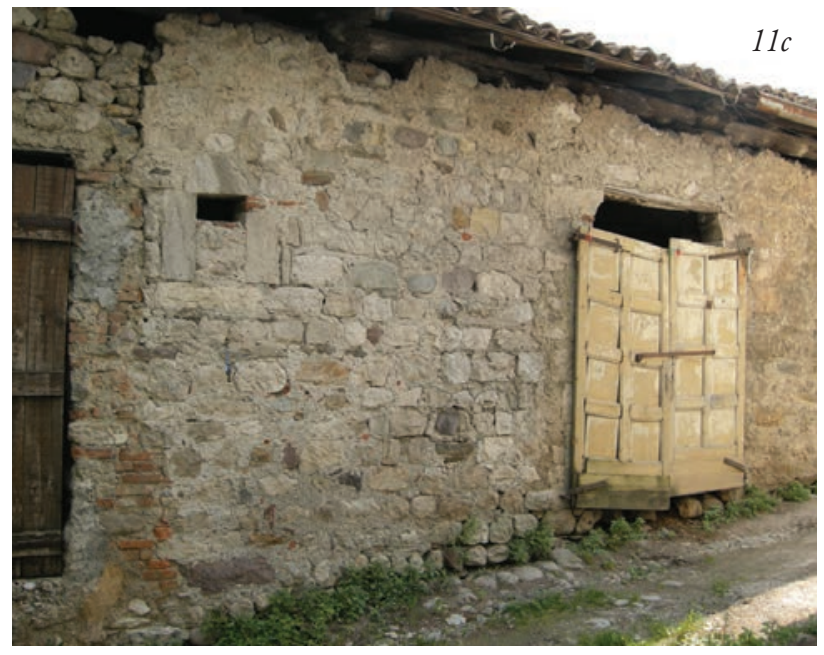

prevalentemente aristocratica secondo la loro qualifica di domine $e^{38}$. L'accesso alla comunità avveniva con la solenne dedicazione a Dio e la professione emessa davanti all'altare, su cui erano poste le offerte del pane e del vino, nelle mani dell'arciprete o di un presbitero della pieve, alla presenza dell'intera comunità e dei familiari. Davanti a loro la professa indossava l'abito religioso, riceveva il bacio di pace e l'abbraccio di fraterna accoglienza da parte della superiora e delle altre sorores, a cui i chierici della matrice attribuivano poi un canonicatum quale vitalizio ${ }^{39}$.

Il caso di Precia è tuttavia più complesso perché la decisione di mettere i vestimenta ecclesiastica e di intraprendere il pellegrinaggio sulla tomba di Pietro fu la conseguenza degli sviluppi giudiziari della sua denuncia. In un primo momento, infatti, l'intenzione dei figli era solo quella di tutelarsi dal ricorso al tribunale cittadino, rinchiudendo la madre in una struttura ecclesiastica protetta e lontano dai clamori; il rischio però del procedimento giudiziale avviato dalla donna davanti al vescovo, li obbligava a scegliere una via più sbrigativa e tale - se possibile - da rendere inoffensivo il ricorso al foro ecclesiastico. I figli allora giocano d'anticipo scegliendo la carta del pellegrinaggio: si recano a Santa Maria con gli officiali della pieve, alcuni vassalli e una cospicua offerta di denaro che depositano sull'altare in cambio della disponibilità a trattenere la madre, che avrebbe così rinunciato all'investitura del patrimonio familiare, edella promessa di ricchezze, «honorem et bonum et magnum averum» fatta alla superiora domina Armelina se avesse accolto Precia in sororem. Di fronte agli indugi di quest'ultima, il presbitero Alberto le ricorda che il suo ingresso era subordinato al trasferimento dei beni promessi $\mathrm{e}$, in caso contrario, sarebbe stata allontanata dalla domus perdendo le garanzie della protezione ecclesiastica ${ }^{40}$.

\footnotetext{
37 L'espressione mette a fuoco il paradosso tra solitudine e forme di vita comune, cfr. C. CABY, Finis eremitarum? Les formes régulières et communautaires de l'érémitisme médiéval, in Ermites de France et d'Italie (XI ${ }^{e}-X V^{e}$ siècles), Actes du colloque organisé par l'École française de Rome à la Certosa di Pontignano (5-7 mai 200o) avec le patronage de l'Université de Sienne, a cura di A. Vauchez, Rome 2003 (Collection de l'École française de Rome, 313), p. 77; ARCHETTI, Singulariter in heremo vivere, pp. 150-154.

$3^{8}$ GuYotjeannin, Les lois du sang, pp. 781-784 passim, 786, 788 .

39 «Al parroco spettava la funzione di ispettore degli eremiti operanti nel territorio di sua giurisdizione; egli aveva anche la facoltà di celebrare la cerimonia della vestizione e di rilasciare la relativa patente, mentre l'ordinario diocesano era tenuto a indagare se il candidato aveva i requisiti religiosi e morali necessari per una vita solitaria» (M. SENSI, Il santesato. Eremiti e comunità rurali, rapporti giuridici e umani, in Ermites de France, p. 370). Per la regolarizzazione canonica dell'esperienza religiosa femminile in questo periodo, con particolare riferimento alle canonichesse, cfr. M. PARISSE, s.v., Kanonissen, in Lexikon des Mittelalters, V, München 1991, pp. 907-908; I. CRusıus, "Santimoniales quae se canonicas vocant". Das Kanonissenstift als Forschunsproblem, in Studien zum Kanonissenstift, a cura di I. Crusius, Göttingen 2001 (Studien zur Germania Sacra, 24), pp. 9-38.

40 GuYotjeannin, Les lois du sang, pp. 783-784.
} 
I fatti a questo punto sembrano seguire un copione già scritto: Precia entra in comunità come conversa e si mette a servizio della chiesa di Cure, facendo scattare il ricorso in appello al vescovo da parte di Armelina, comunicato alle autorità cittadine presso la pieve di Val Renovata. Ciò poneva all'angolo in modo definitivo la giustizia civile e invano il messo del podestà si sarebbe recato a Montisola per portare con sé la donna o per notificarle l'atto di citazione del giudice comunale ${ }^{41}$. Essa era ormai sotto la protezione ecclesiastica, come confermano le parole di Casarius, uno dei figli: «Se mia madre sta lassù e si convince ad indossare gli abiti religiosi, quando i funzionari del podestà verranno e la troveranno vestita in quel modo, il giudice del comune non si intrometterà più in questa faccenda»e, aggiungeva circa l'accoglienza della madre nella comunità: «Se Dio e santa Maria porranno in cuore a mia madre di restare lassù, io sarò felice e liberamente darò il consenso affinché lo faccia; e se, a Dio piacendo, starà qui riceverete onore, beni e un grande lascito, grazie a cui si arricchirà questa comunità (domus) $»^{4^{2}}$; mentre l'imputata, una volta indossati gli abiti sacri, non esitò a respingere le garanzie legali datele dalle istituzioni comunali: «Riguardo a questa faccenda - disse - non mi proclamo del podestà di Brescia, ma del signor vescovo e secondo le sue disposizioni dei chierici della pieve; ciò che il vescovo vorrà da me al riguardo io lo farò»43 (figg. 11a, 11b, 11c).

Alla vigilia della festa della pasqua dell'Epifania, cioè il 5 gennaio 1182, dopo aver preso accordi precisi, Casarius accompagnò con i suoi vassalli la madre in riva al lago alla pieve della Val Renovata e da qui con il chierico Pietro a Montisola, dove le venne data ospitalità nella domus di Santa Maria de Cora; in seguito la somma di venti lire, una pelle di agnello e una pezza di pignolato furono il prezzo della sua investitura per l'ingresso in comunità sancito da una solenne liturgia e dalla messa cantata in onore della Trinità ${ }^{44}$. Furono Casarius, Boxadrus, Urso e il nipote Caponino a farsene carico, i quali dissero alla donna il giorno della professione, incoraggiandola: "Così potete aiutare noi e voi. Andate avanti e ponete l'investitura sull'altare di Santa Maria, fatelo per voi e per le vostre preoccupazioni», e così avvenne. Poi fu abbracciata e baciata dai presenti, mise le sue mani in quelle del presbitero Alberto in segno di sottomissione e questi la accolse tra le suore della casa, la baciò e le rammentò la promessa di cospicui beni che lei aveva fatto alla comunità, senza i quali sarebbe venuta meno la protezione ecclesiastica che le assicurava la residenza a Cure ${ }^{45}$.

\section{RIFLESSI SULLA MOBILIT $\square$ LOCALE}

La forzata reclusione di Precia mette in luce la complessità dei rapporti tra i gruppi aristocratici e le istituzioni ecclesiastiche, quali interlocutori privilegiati e obbligati di una reciprocità di "servizi" espressi secondo le modalità dei legami feudo-vassallatici e signorili: aiuto e consiglio innanzitutto, protezione, prestazione di buoni offici, interscambio di beni e diritti di elevato valore. In questa non
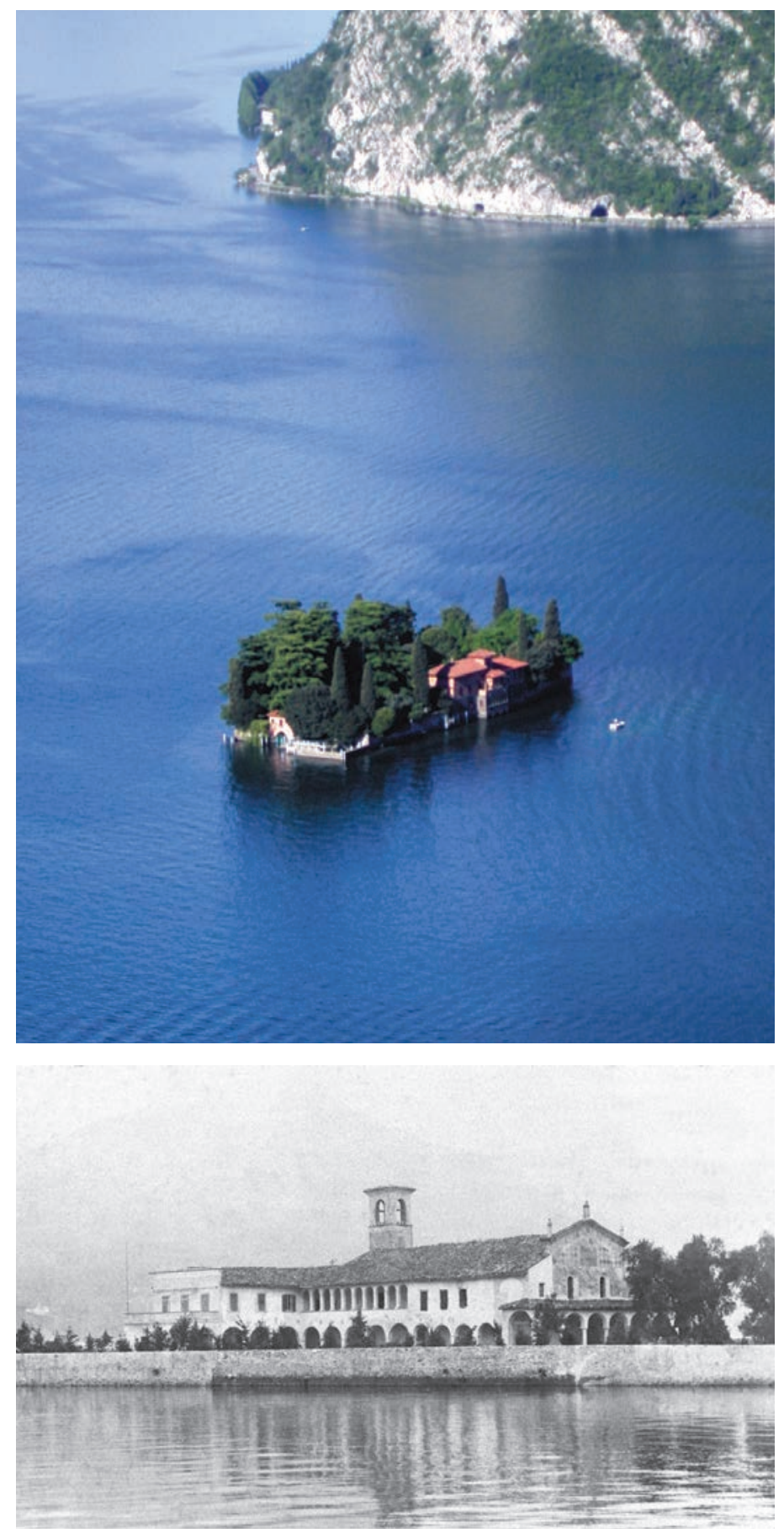

Figg. 12a, 12b-Isoletta di San Paolo nel lago d'Iseo, già sede di una cella cluniacense e poi di un convento francescano (nella foto storica).

sempre limpida né chiara dialettica sono i laici a muoversi con maggiore disinvoltura, badando senza troppi scrupoli al raggiungimento del loro scopo, mentre i loro interlocutori cercano a fatica di riservare una qualche forma di autonomia al potere ecclesiastico - Precia è accolta dietro cessione dell'investitura e Bontat è chiamata a rispondere sui rischi di ritorsioni economiche a danno di Santa Maria del Giogo - giocando «sul doppio tavolo di una Chiesa nel secolo e

${ }_{41}$ All'arrivo del ministeriale cittadino a Santa Maria a Cure, Precia non esitò a dire che «non era tenuta a dare né auxilium né consilium», perché nella questione riguardante sua figlia Jacobina e suo genero Milo non rispondeva all'autorità podestarile, ma a quella ecclesiastica a cui era stato fatto appello e nella quale si riconosceva (Ibidem, p. 784).

${ }^{42}$ Ibidem, p. 783 .

${ }^{43}$ Ibidem, p. 784 .

${ }^{44}$ Ibidem, pp. 786, 781-784 passim.

${ }_{45}$ Ibidem, pp. 783, 782. 


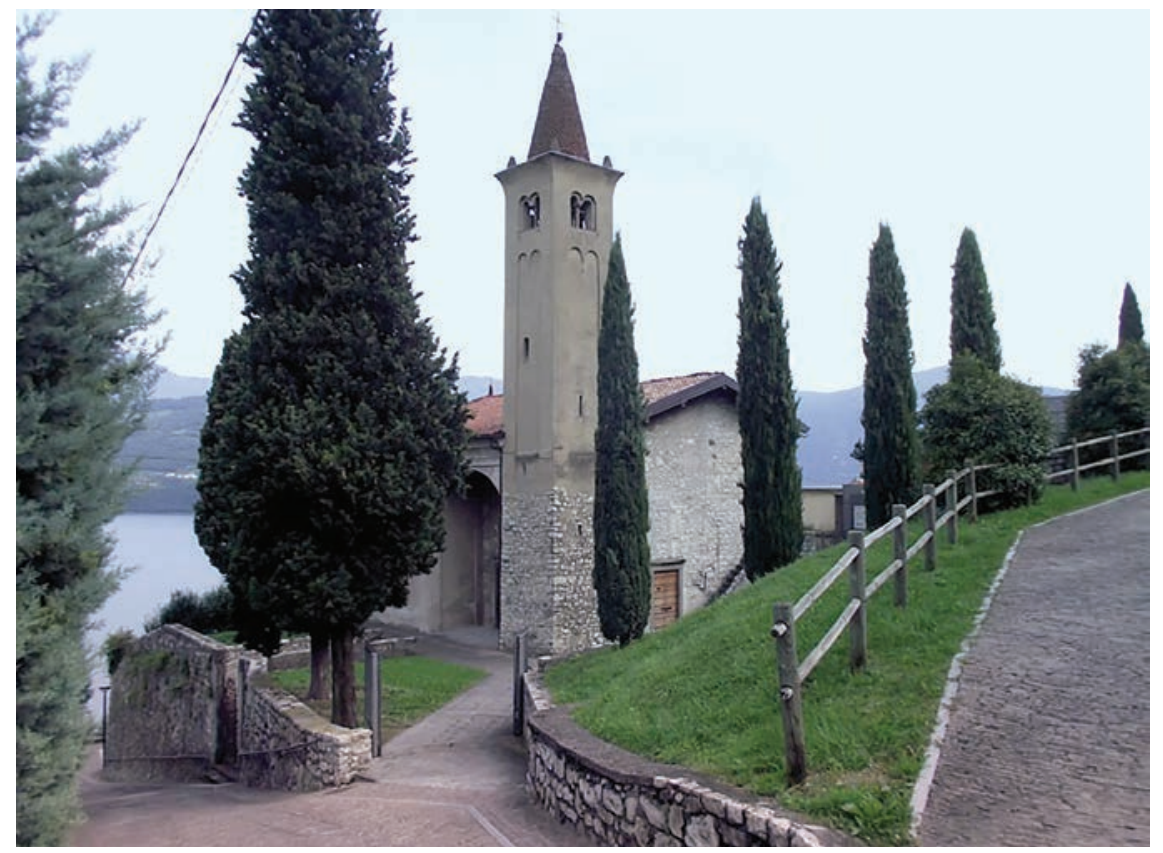

Fig. 13 - Tavernola Bergamasca (Bergamo), sul lago d'Iseo, chiesa di San Pietro.

mostra subito accondiscendente: «Liberamente parlerò io con il vescovo, se verrà a San Paolo, perché lui conosce bene la mia questione», denunciando che i figli avrebbero fatto di tutto per impedire l'incontro ${ }^{8}$ (figg. 12a, 12b). Per questo, in attesa di maggiori garanzie legali, trova temporaneo rifugio in territorio bergamasco, dove resta per alcuni giorni ospite della chiesa di San Pietro di Tavernola (fig. 13).

Lo stesso fa Armelina, conversa di Santa Maria per seguire la vicenda: attraversa il lago sia verso la sponda bresciana, sia verso quella bergamasca, si reca alla curia vescovile di Pisogne, si sposta a San Pietro di Tavernola, va al porto di Iseo e in episcopio a Brescia, mentre alla pieve della Val Renovata comunica al messo del podestà di aver fatto appello al vescovo. Ancora, Precia partecipa a Rodengo alle esequie del marito e si reca dalla conversa Bontà a Santa Maria de Zuvo pregandola di andare da sua figlia per portarle «una camicia, due tazze e un vestito di panno grezzo, che fa recapitare da Fiducia di

al di sopra di esso» ${ }^{46}$. Appare altresì innegabile l'influsso di queste "recluse" sulla società circostante, da cui erano separate da strutture claustrali di tipo monastico, ma con la quale restavano in contatto per molteplici necessità spirituali e materiali ${ }^{47}$. La loro condizione di isolamento presso una chiesa o un monastero poteva essere temporanea e confluire solo in un secondo momento in una forma stabile all'interno della comunità per motivazioni sociali e di sicurezza, oltre che religiose. Ciò avveniva con il consenso e l'approvazione delle autorità ecclesiastiche e senza perdere i contatti con il mondo esterno, dove all'occorrenza si potevano recare con grande libertà di movimento e senza particolari restrizioni, come conferma l'intricata vicenda di Precia.

La dinamica delle relazioni vede i protagonisti muoversi con estrema facilità da un luogo all'altro della diocesi e fuori di essa per dare seguito ai loro affari. Ne emerge una geografia di spazi, centri di potere, itinerari e edifici sacri di notevole rilevanza che, in taluni casi, rappresenta anche la loro prima attestazione documentaria scritta. Le persone si spostano dalla città per raggiungere il priorato di Rodengo in occasione della sepoltura di Baxacanus, come pure dai luoghi vicini (Ome, Rodengo, Polaveno, Iseo, ecc.); il messo comunale si reca da Brescia a Sale Marasino e a Montisola per notificare la convocazione a Precia e, quest'ultima, lascia la sua casa di Ome per andare a Cure e da qui a Pisogne, a Brescia e a Tavernola Bergamasca per incontrare il vescovo o un suo delegato; alla richiesta del presule di incontrarla si

Inzino»49. I suoi figli vanno in casa sua ea Montisola, curano i loro interessi in città, fanno arrivare merci a Iseo, si imbarcano a Sale Marasino e li troviamo a Rodengo, come pure Caponino, in continuo movimento per seguire i loro interessi a Ome, in Franciacorta e in città. La conversa di Santa Maria del Giogo, domina Bontat, si porta con la monaca Flora e il converso Ugolino all'abitazione di Milo e Jacobina: «Domina Precia mi manda qui - dice loro - perché vuole venire a stare con voi, se a voi sta bene», e questi di rimando: «Certamente, la cosa ci piace molto». E lei: «Domina Precia vuole essere sicura che verrà trattata bene e onorevolmente», mentre Milo giurando le risponde: «Se vuole abitare con noi stabiliamo che sia padrona e signora dei miei e dei suoi averi. Se vuole stare da sola sulla sua tenuta e conserverà l'eredità di sua figlia, voglio assicurarle ogni anno un vitalizio di quattro lire di denari, quattro carri di vino, quattro galete di olio, dodici minali di cereali e due minali di legumi» ${ }^{\circ}$.

A fronte del recupero dell'eredità la donna aveva la garanzia che sarebbe stata trattata come «donnam et majorem honorifice», cioè riconosciuta per il ruolo signorile di anzianità che aveva all'interno del gruppo familiare; in caso contrario il giuramento sarebbe stato privo di valore, come ricorda Fiducia di Inzino. Il teste riferì poi le parole del converso Ugo presente all'incontro: «Io sono il messo di Precia che, come sembra, è una delle donne più infelici al mondo e ha agito male riguardo all'eredità di Baxacaponus, che doveva invece spettare a sua figlia Jacobina. Per questo

\footnotetext{
${ }^{46}$ Ibidem, p. 771.

${ }_{47}$ Sull'eremitismo femminile, cfr. E. PászTor, Ideali dell'eremitismo femminile in Europa tra i secoli XII-XV, in Eremitismo nel francescanesimo medievale, Atti del XVII Convegno internazionale (Assisi, 12-14 ottobre 1989), Perugia 1991 (Società internazionale di studi francescani. Convegni, 7), pp. 129-164 [ora anche in EAD., Donne e sante. Studi sulla religiosità femminile nel medioevo, Roma 2000 (Religione e società, 37), pp. 65-96]; G. CASAGRANDE, Forme di vita religiosa femminile solitaria in Italia centrale, in Eremitismo nel francescanesimo, pp. 66-74; A. BENVENUTI PAPI, "In castro poenitentiae". Santità e società femminile nell'Italia medievale, Roma 1990 (Italia sacra, 45), specie le pp. 305-402; A. ALbUZzI, Il monachesimo femminile nell'Italia medioevale. Spunti di riflessione e prospettive di ricerca in margine alla produzione storiografica degli ultimi trent'anni, in Dove va la storiografia monastica in Europa? Temi e metodi per lo studio della vita monastica e regolare in età medievale alle soglie del terzo millennio, Atti del Convegno internazionale (Brescia-Rodengo, 23-25 marzo 2000), a cura di G. Andenna, Milano 2001, 131-189 passim; DAL PINO, Eremitismo libero, pp. 380, 394-395.

${ }^{48}$ Guyotjeannin, Les lois du sang, p. 781.

$49 \mathrm{Ibidem}$, p. 786, la testimonianza viene escussa il venerdì 16 luglio 1182, ma l'incontro e le trattative erano intercorse un anno prima, quando Precia era salita a Santa Maria del Giogo sopra Sulzano nella festa di San Giacomo (25 luglio).

50 Ibidem, p. 786.
} 


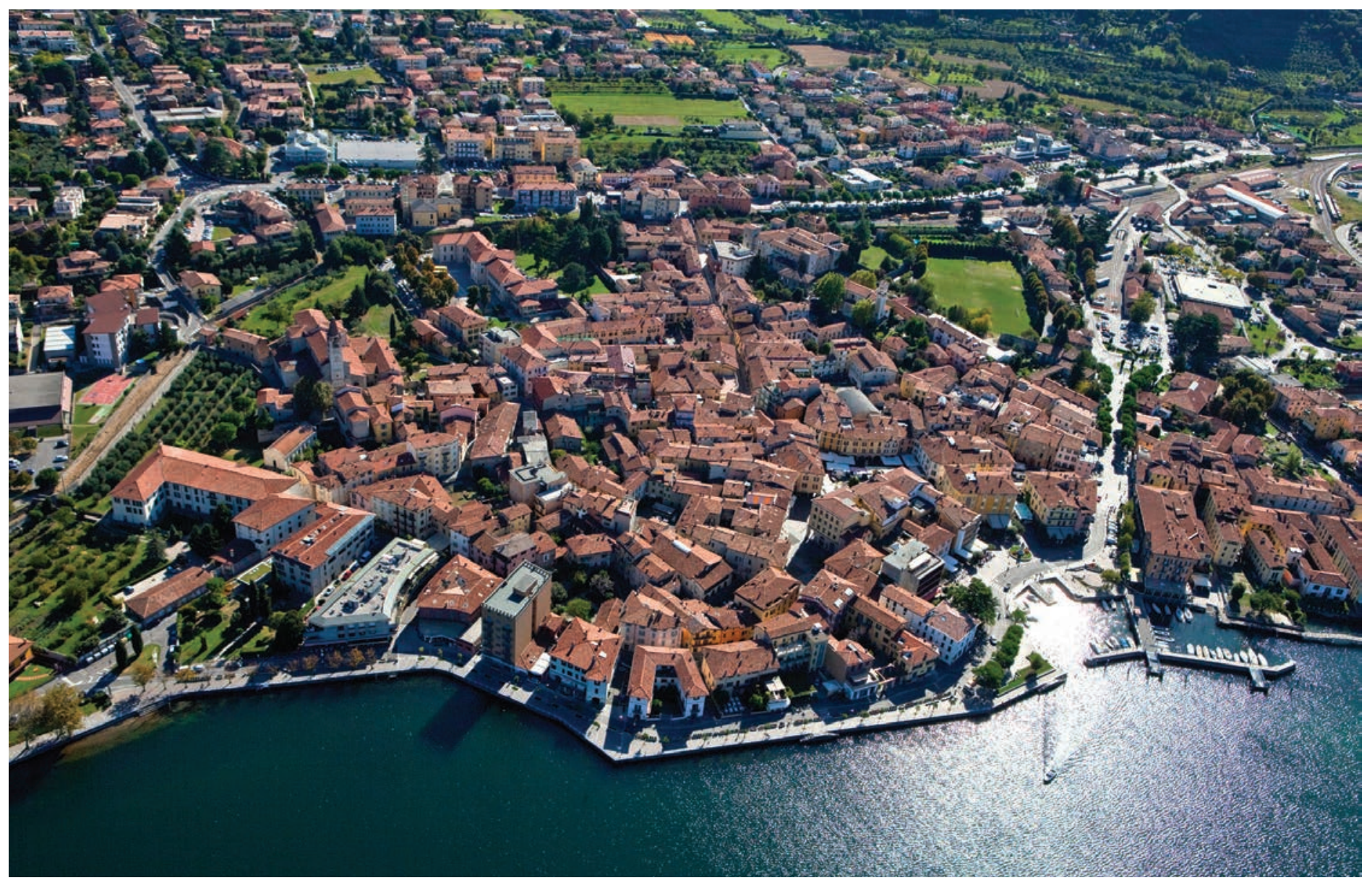

Fig. 14 - Veduta del borgo di Iseo (Brescia), nel medioevo sede della curia episcopale e della pieve di Sant'Andrea.

giuro e per la sua volontà stabilisco di farla venire a stare con voi e di dare a Jacobina il patrimonio di Baxacaponus, che le deve appartenere. E lei ha confermato che Caponino non è figlio suo». Milo e la moglie assicurarono che avrebbero mantenuto fede alle loro parole e, fatta portare dell'acqua in un catino - nella stanza in cui si trovavano e dove il miles degli Ugoni era a letto a causa del dolore ad una gamba -, si lavarono entrambi le mani e la faccia come per sciogliersi dalle conseguenze derivanti dagli obblighi della loro promessa qualora Precia non avesse rispettato i suoi doveri nei confronti della figlia ${ }^{11}$ (fig. 14).

D'altra parte, se la cattedrale di San Pietro de Dom è il centro dell'azione episcopale in città, dove alcuni dei protagonisti sono registrati a discutere della vicenda, il priorato cluniacense di Rodengo è attestato per la funzione cimiteriale e ricevere le spoglie del defunto Baxacaponus, mentre la piccola cella cluniacense sull'isola di San Paolo, in mezzo al lago d'Iseo, è menzionata per il suo isolamento come possibile luogo di incontro tra Precia e il vescovo nel suo trasferimento dalla curia di Pisogne a quella di Iseo verso la città ${ }^{2}$. Le chiese di Santa Maria de Cora di Montisola e di San Cassiano di Martignago a Sulzano rientrano nella giurisdizione ecclesiastica della pieve di San Zenone di Sale Marasino, indicata come plebs Vallis Renovate, come pure la chiesa alpestre di Santa Maria del Giogo sopra Sulzano, oggi in territorio di Polaveno, mentre le pievi di Santa Maria di Pisogne e di Sant'Andrea di Iseo appartenevano alla mensa vescovile ed erano sede amministrativa di una curia episcopale53; di San Pietro di Tavernola, in territorio bergamasco, restano ancora nell'apparato murario i segni della sue antiche origini romaniche, che trovano conferma nelle deposizioni testimoniali (figg. 15, 16). I tracciati stra-

\footnotetext{
${ }^{51}$ Ibidem, pp. 787-788. Fiducia di Inzino rilascia la sua deposizione giurata il 27 giugno 1182.

${ }_{52}$ Per uno sguardo generale, cfr. Visita apostolica e decreti di Carlo Borromeo alla diocesi di Brescia, I. La città, a cura di A. Turchini, G. Archetti, Brescia 2003 (Brixia sacra, VIII, 1-2), pp. 85-90, 107-119 (San Pietro de Dom); Visita apostolica e decreti, III, pp. 72, 75 (San Paolo del lago); Visita apostolica e decreti di Carlo Borromeo alla diocesi di Brescia, V. Valle Trompia, Pedemonte e Territorio, a cura di A. Turchini, G. Archetti, Brescia 2005 (Brixia sacra, X, 1-2), pp. 51-54 (Rodengo); Il Duomo Nuovo di Brescia, 1604-2004. Quattro secoli di arte, storia, fede, a cura di M. Taccolini, Brescia 2004; San Nicolò di Rodengo. Un monastero di Franciacorta tra Cluny e Monte Oliveto, cit.; A. Archetti, San Paolo del lago. Notizie storiche, leggende, curiosità di un'isola del lago d'Iseo, Brescia 2003 (Terre bresciane); G. ARchetтI, Servire Dio in santità e giustizia. Da Cluny alla Franciacorta, in Monachesimo e sviluppo del territorio nelle Alpi lombarde, a cura di O. Franzoni, Breno 2005, pp. 223-267; ID., Fraternità, obbedienza e carità. Il modello cluniacense, in A servizio del Vangelo, 1. L'età antica e medievale, pp. 483-513, 654-659.

53 Si rimanda a GUERRINI, Per la storia dell'organizzazione ecclesiastica, pp. 128-129; ID., La pieve di Sale Marasino, pp. 3-17; Visita apostolica e decreti, III, pp. 41-49, 66, 68, 76, 81-82; Storia ed arte nella chiesa di San Zenone, pp. 17-25; ARcheTTI, Berardo Maggi, pp. 215-221, 335-344, 507-539; ID., La fede e l'aratro. Pievi e cura delle anime nel medioevo in Franciacorta, in Pievi della montagna lombarda, a cura di O. Franzoni, Brescia-Breno 2006, pp. 233-275; Società bresciana e sviluppo del romanico, cit., passim; G. ARchetтI, San Vigilio e la pieve di Iseo. Note storiche per lo studio di una Chiesa locale nel Medioevo, Pubblicazione a cura della Biblioteca Comunale "Fra Fulgenzio Rinaldi" di Iseo, Brescia 2007; Storia ed arte nella chiesa di San Zenone a Sale Marasino, pp. 17-25; A. Bianchi, A. Macario, In loco de Pisoneis. Pisogne 1299: il borgo del vescovo, con contributi di G. Medolago e R. Vangelisti, Comune di Pisogne (Bs) 2008; A servizio del Vangelo, cit.
} 


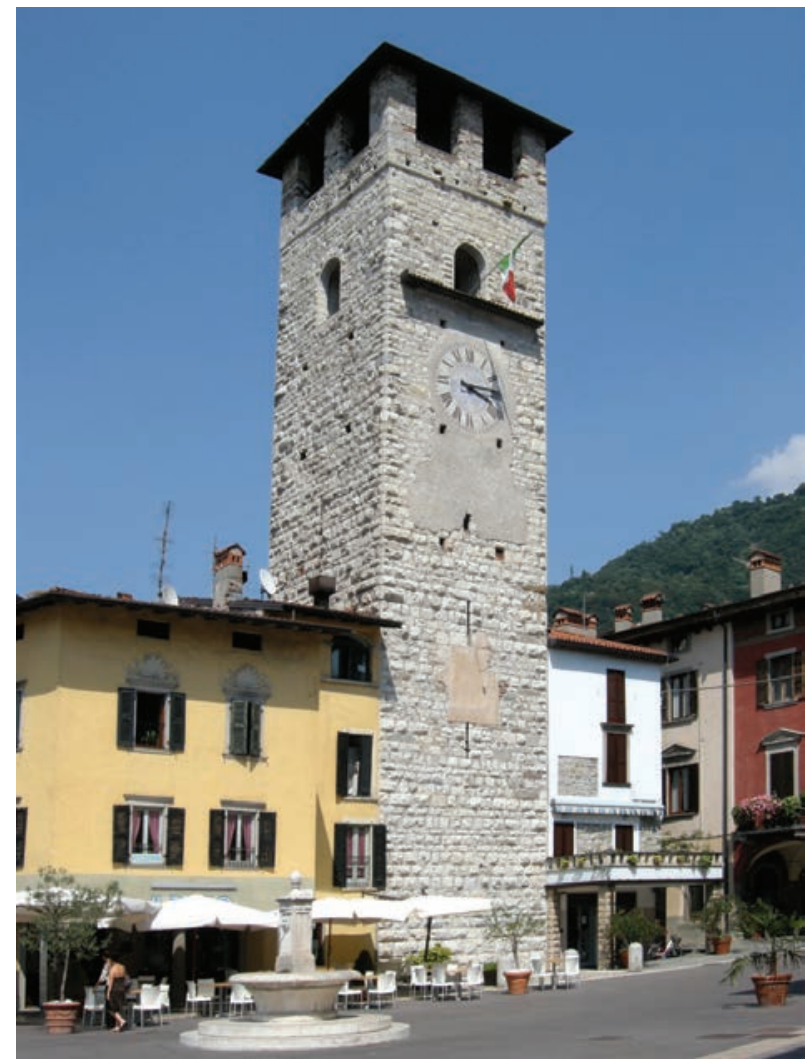

Fig. 15-Pisogne (Brescia), la torre medievale del vescovo centro della curia episcopale.

dali invece si snodano tra la città, la Franciacorta e la bassa Valcamonica per completarsi con i porti e le rotte lacuali del Sebino a Iseo, Sulzano, Sale Marasino, Montisola, San Paolo del Lago, Tavernola e Pisogne, ecc.

\section{IN VIAGGIO VERSO LA TOMBA DI PIETRO}

Con espressioni affettuose come «cara mater», «pulcra mater», «vi imploro» o «vi prego», Precia alla fine viene prima blandita e poi "convinta" a fare voto di partire in pellegrinaggio con uno dei figli, come ricorda un teste riferendo le parole di Pietro: «Cara mater, il primo bene che abbiamo inizia dal padre e dalla madre. Io perciò ho fatto voto di andare a San Pietro a Roma per le grandi angustie e le preoccupazioni in cui mi trovavo. Con me ha fatto voto anche mia moglie e intendo portare un mio figlio; ma dal momento che so che avete fatto voto di andare a San Pietro, desidero portarvi io, giacché è meglio per voi che veniate con me piuttosto che con un'altra persona. Pulcra mater, non ho che una sorella. Credete che non le voglia bene? Io la amo quanto voi. E spero in Dio che così si possa tornare ad essere in pace e amici» 54 . Le relazioni con i figli tuttavia e il pasticcio del patrimonio, alla fine, restavano il vero cruccio della donna anche nell'isolamento di Montisola, come si apprende dalle parole di Armelina: «Domina, adesso siete dedicata a Dio e a santa Maria. Ora dovete dirmi la verità. Per Dio, non rimanete più in questa angoscia, per non offendere il Signore e il vescovo non se la prenda con noi per questo. Quale persona dovete amare di più della vostra Jacobina?». E quella rispondeva: «Non vi è persona alcuna che non ami

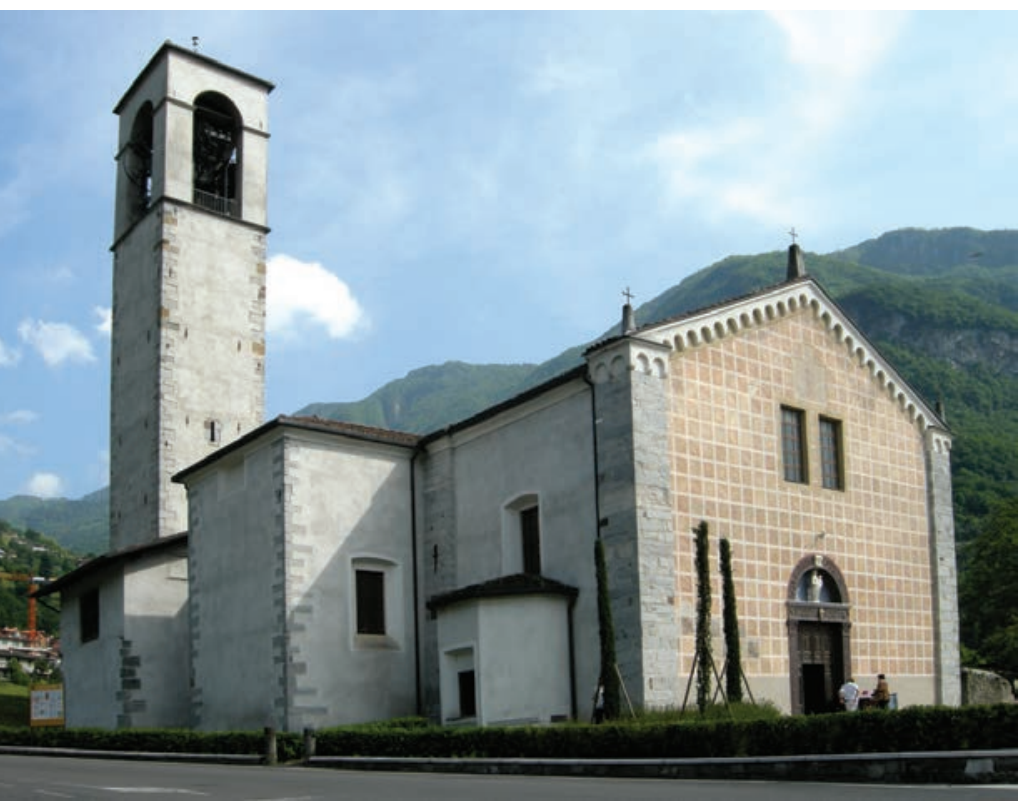

Fig. 16-Pisogne (Brescia), pieve di Santa Maria.

più di lei, e sua deve essere l'eredità. Per Dio, Caponino non è mio figlio, né è uscito dal mio corpo. Davvero una madre stolta è colei che abbandona suo figlio, ed è una fortuna che non sia morto» ${ }^{55}$.

Il pensiero dei figli, dunque, tra timore e affetto materno risulta essere il sentimento dominante sempre accompagnato dal timore che possa succedere loro qualcosa di grave, e in questo ne risulta inficiata la sua stessa difficile quanto tardiva confessione. Inoltre, mentre sembra sinceramente amareggiata che Jacobina sia stata esclusa dall'eredità, Precia manifesta sentimenti di ben altro tenore nei confronti di Caponinus. A lui - iniziale vittima innocente divenuto denuncia vivente dell'inganno e causa delle sue disavventure una volta saputo dell'imbroglio - riserva espressioni molto dure: «Il diavolo me lo ha gettato addosso!», ripetendo che non era suo figlio ma suo nipote, essendo nato dalla moglie del suo primogenito: «Dio maledica Obizo e i suoi figli!» ${ }^{56}$. Al contrario, verso Jacobina si avverte premurosa attenzione, documentata anche dai doni che gli fa avere per mezzo di Bontà di Santa Maria del Giogo e del messo Fiducia di Inzino: unam camisiam, duas scuthellas et unum causape.

Nonostante la denuncia della donna e l'intenzione di giungere alla verità, le cose hanno uno sviluppo diverso, come si apprende da domina Armelina: «Se avete questa ferma volontà, come dite, perché non la portate avanti affinché si conosca la verità? Se non volete andare a Brescia, avete comunque amici a cui affidare tale compito». E Precia rispondeva: «Se vi fosse persona a cui dare questo compito, $\mathrm{i}$ miei figli non si fermerebbero neppure di fronte all'omicidio. Voglio bene a loro e a mia figlia Jacobina; sono preoccupata che gli possa accadere qualcosa di male e sono rattristata se Jacobina perderà l'eredità di suo padre Baxacaponus». E proseguiva: «Cara domina, come non posso essere infelice e addolorata per quel diavolo bilioso, che il demonio ha fatto nascere, che sperpera il mio patrimonio e quello di mia figlia Jacobina; come dovremmo noi godere! Poiché, da quando è venuto a saperlo, non mi vuole più bene, ma ha sempreavuto

\footnotetext{
54 Guyotjeannin, Les lois du sang, p. 784.

55 Ibidem, p. 782.

${ }^{5}$ Ibidem, p. 785 .
} 
odio?»57. Conoscendo bene l'inclinazione materna i domini di Salò seppero sfruttare a loro vantaggio la sua debolezza, con calcolata maestria, usando un accurato dosaggio di brutalità e di scaltrezza tanto nel comportamento quanto nelle parole; lo si vede quando uno di loro domanda alle religiose di Santa Maria di accoglierla, coprendosi dietro l'ispirazione divina o quando senza mezzi termini le dicono che può ritirarsi in solitudine perché ormai «ha visto abbastanza le cose di questo mondo» e con dolci parole la scongiurano di non disonorarli: «Vi prego, cara madre, in nome di Dio, non dovete nos desonorare nec destruere $\aleph^{8}$.

La vigilia della festa della Purificazione di Maria, il primo giorno del mese di febbraio 1182, secondo la testimonianza di Dulcera, conversa di Santa Maria de Cora, Casarius e Pietro da Salò giunsero a Montisola dicendo che avevano deciso di andare in pellegrinaggio a San Pietro a Roma con la loro madre ${ }^{59}$. La conversazione avvenne nella sala riscaldata col fuoco posta vicino alla chiesa, presenti il prete Alberto e altri familiari della domus. L'epilogo era vicino e non si poteva tornare indietro. In un ultimo tentativo di sfuggire al suo destino, «Domina Precia - riferì Armelina - giurò di portarmi a Roma a sue spese. "Voglio che lei mi porti con sé e senza di lei non devo andare"», e Pietro rispose: «A noi sta bene. Preparate i bagagli!» ${ }^{60}$. A cui seguirono le domande sul viaggio, sulla partenza e su quando si doveva andare. Che cosa sia accaduto dopo a Precia, però, la teste disse di non saperlo perché non la vide più: «quia postea eam non vidit», né ebbe modo di accompagnarla lungo il tragitto romeo. Itinerario lungo e pericoloso che molti intraprendevano, ma che non tutti riuscivano a ultimare, parecchi poi si perdevano per via, mentre altri si ammalavano e spesso morivano...

Temeva dunque a tal punto la loro vendetta da preoccuparsi per la sua incolumità fisica e, lasciata la sicura quiete del ricetto isolano al seguito dei familiari, le sue tracce si perdono proprio all'inizio del cammino verso la tomba dell'apostolo Pietro, dove probabilmente la raggiunse la quiete à serenità eterna dando finalmente un po' di pace al suo animo inquieto.

\footnotetext{
57 Ibidem, p. 785.

${ }^{8}$ Ibidem, p. 784.

59 Ibidem, p. 782, ciò è confermato anche da domina Armelina (ivi, p. 784).

6o Ibidem, pp. 784 e 785 per la citazione successiva.
}

* Referenze fotografiche: Archivio di "Brixia sacra”, Associazione archeologica USPAAA, Angelo Valsecchi, Gabriele Archetti, Orione. Cultura, lavoro, comunicazione. 